\title{
EXTRACTING ANGULAR DIAMETER DISTANCE AND EXPANSION RATE OF THE UNIVERSE FROM TWO-DIMENSIONAL GALAXY POWER SPECTRUM AT HIGH REDSHIFTS: BARYON ACOUSTIC OSCILLATION FITTING VERSUS FULL MODELING
}

\author{
Masatoshi Shoji, Donghui Jeong, and EiIChiro Komatsu \\ Department of Astronomy, University of Texas at Austin, 1 University Station, C1400, Austin, TX 78712, USA; mshoji@astro.as.utexas.edu \\ Received 2008 May 27; accepted 2008 October 20; published 2009 March 9
}

\begin{abstract}
We present a method for extracting the angular diameter distances, $D_{A}$, and the expansion rates, $H$, of the universe from the two-dimensional baryon acoustic oscillations (BAO) in the galaxy power spectrum. Our method builds upon the existing algorithm called the "fit-and-extract" (FITEX) method, which allows one to extract only $D_{A}^{2} / H$ from a spherically averaged one-dimensional power spectrum. We develop the FITEX-2d method, an extension of the FITEX method, to include the two-dimensional information, which allows us to extract $D_{A}$ and $H$ simultaneously. We test the FITEX-2d method using the Millennium Simulation as well as simplified Monte Carlo simulations with a bigger volume. The BAOs, however, contain only a limited amount of information. We show that the full modeling, including the overall shape of the power spectrum, yields much better determinations of $D_{A}$ and $H$, hence the dark energy equation of state parameters such as $w_{0}$ and $w_{a}$, than the BAO-only analysis by more than a factor of 2, provided that nonlinear effects are under control.
\end{abstract}

Key words: cosmology: theory - large-scale structure of universe

\section{INTRODUCTION}

Dark energy, discovered via the observed luminosity distances out to high- $z$ Type Ia supernovae (Riess et al. 1998; Perlmutter et al. 1999), is the most mysterious element in physics today (see Copeland et al. 2006 for a recent review).

As dark energy primarily affects the expansion rate of the universe, one can gain information on the nature of dark energy by measuring the cosmological distances as well as the expansion rates of the universe accurately. ${ }^{1}$

While the cosmic microwave background (CMB) and the Type Ia supernovae can be used for measuring the angular diameter distance out to $z \simeq 1090$ and the luminosity distances out to $z \lesssim 2$, respectively, the power spectrum of matter distribution in the universe can be used to measure the angular diameter distances as well as the expansion rates of the universe out to a wider range of redshifts.

Two length scales are encoded in the matter power spectrum, $P(k)$ (see, e.g., Weinberg 2008):

1. The comoving Hubble horizon size at the matter-radiation equality, $r_{H}\left(z_{\mathrm{eq}}\right)=c /\left[a\left(z_{\mathrm{eq}}\right) H\left(z_{\mathrm{eq}}\right)\right]$.

2 . The comoving sound horizon size at the so-called drag epoch at which baryons were released from photons, $r_{s}\left(z_{\mathrm{drag}}\right)=\int_{0}^{t\left(z_{\mathrm{drag}}\right)} d t c_{s}(t) / a(t)$, where $c_{s}(t)=c /[\sqrt{3}(1+$ $\left.\left.a(t) 3 \Omega_{b} /\left(4 \Omega_{\gamma}\right)\right)\right]$ is the sound speed of photon-baryon fluid.

The former determines the overall shape of the power spectrum of dark matter including the location of the peak of $P(k)$ at $k_{\text {eq }} \equiv 1 / r_{H}\left(z_{\text {eq }}\right)$, whereas the latter determines the location of the baryonic features called the baryon acoustic oscillations (BAOs).

These length scales can be predicted from the five-year data of the Wilkinson Microwave Anisotropy Probe (WMAP)

\footnotetext{
1 While dark energy also affects the growth rate of the amplitude of matter fluctuations, which has been seen in the data via the so-called integrated Sachs-Wolfe (ISW) effect (e.g., Boughn \& Crittenden 2004; Nolta et al. 2004; Afshordi et al. 2004; Fosalba et al. 2003; Fosalba \& Gaztañaga 2004), we do not discuss the effect on the amplitude of fluctuations in this paper.
}

(Hinshaw et al. 2009; Dunkley et al. 2009; Komatsu et al. 2009)²:

$$
\begin{aligned}
k_{\mathrm{eq}} \equiv \frac{1}{r_{H}\left(z_{\mathrm{eq}}\right)} & =(0.968 \pm 0.046) \times 10^{-2} \mathrm{Mpc}^{-1}, \\
r_{s}\left(z_{\mathrm{drag}}\right) & =153.3 \pm 2.0 \mathrm{Mpc},
\end{aligned}
$$

and

$$
z_{\text {eq }}=3176_{-150}^{+151}, \quad z_{\text {drag }}=1020.5 \pm 1.6 .
$$

These lengths can be used as the "standard rulers," which give us the angular diameter distances as well as the expansion rates of the universe (Seo \& Eisenstein 2003; Blake \& Glazebrook 2003; Hu \& Haiman 2003). ${ }^{3}$

We, as observers who measure the angular and redshift distribution of galaxies, can measure four distance ratios given by

$$
\begin{aligned}
\theta_{\mathrm{eq}}(z) & =\frac{r_{H}\left(z_{\mathrm{eq}}\right)}{(1+z) D_{A}(z)}=\frac{1}{k_{\mathrm{eq}}(1+z) D_{A}(z)}, \\
\theta_{S}(z) & =\frac{r_{s}\left(z_{\mathrm{drag}}\right)}{(1+z) D_{A}(z)}, \\
\delta z_{\mathrm{eq}}(z) & =\frac{r_{H}\left(z_{\mathrm{eq}}\right) H(z)}{c}=\frac{H(z)}{k_{\mathrm{eq}} c}, \\
\delta z_{s}(z) & =\frac{r_{s}\left(z_{\mathrm{drag}}\right) H(z)}{c},
\end{aligned}
$$

where $D_{A}(z)$ is the proper (i.e., not comoving) angular diameter distance. We measure $\theta_{\mathrm{eq}}(z)$ and $\theta_{s}(z)$ by comparing the

\footnotetext{
2 These predictions assume a flat universe and dark energy being the vacuum energy. For a nonflat universe with dark energy having a constant equation of state, $w$, the WMAP five-year data yield $k_{\text {eq }}=\left(0.975_{-0.045}^{+0.044}\right) \times 10^{-2} \mathrm{Mpc}^{-1}$, $r_{s}\left(z_{\text {drag }}\right)=153.4_{-2.0}^{+1.9} \mathrm{Mpc}, z_{\text {eq }}=3198_{-146}^{+145}$, and $z_{\text {drag }}=1019.8 \pm 1.5$. 3 The matter power spectrum also contains the third distance scale, the Silk damping scale, which can also be used as the standard ruler. The Silk damping scale is the smallest of these three distance scales, and its effect (i.e., the suppression of power below the Silk damping scale) is not as prominent as the effects of the other two distance scales. Nevertheless, the Silk damping must be taken into account when we model the full shape of the power spectrum.
} 
predicted lengths with the corresponding observed lengths perpendicular to the line of sight, and $\delta z_{\mathrm{eq}}(z)$ and $\delta z_{s}(z)$ from the lengths parallel to the line of sight. ${ }^{4}$

The BAOs have been detected in the current galaxy redshift survey data from the Sloan Digital Sky Survey (SDSS) and the Two-degree Field Galaxy Redshift Survey (2dFGRS) (Eisenstein et al. 2005; Cole et al. 2005; Hütsi 2006; Percival et al. 2007). However, the current data are not yet sensitive enough to yield $D_{A}(z)$ and $H(z)$ separately (Okumura et al. 2008); thus, one can only determine a combined distance scale ratio from the spherically averaged power spectrum. Since two spatial dimensions are available on the sky and one dimension is available along the line of sight, one can measure

$$
\left[\theta_{s}^{2}(z) \delta z_{s}(z)\right]^{1 / 3}=\frac{r_{s}\left(z_{\mathrm{drag}}\right)}{\left[(1+z)^{2} D_{A}^{2}(z) c / H(z)\right]^{1 / 3}} .
$$

Eisenstein et al. (2005) have measured this quantity at $z=0.35$ from the SDSS Luminous Red Galaxies (LRG), and Percival et al. (2007) have extended their analysis to include more data from the SDSS LRG, as well as the SDSS main galaxy samples and the 2dFGRS galaxies at $z=0.2$.

Komatsu et al. (2009) have combined these measurements with the CMB distance ratios determined from the WMAP fiveyear data, the "WMAP distance priors," to obtain the constraints on dark energy properties. The analysis performed in Komatsu et al. (2009) is a proto-type of what one can do in the future. It is clear that we can gain more information if we can measure $D_{A}(z)$ and $H(z)$ simultaneously at various redshifts. Therefore, in the future we should be able to perform a much more sensitive test of dark energy properties by combining $D_{A}(z)$ and $H(z)$ from the future galaxy survey data, and the CMB distance priors from the future CMB experiments such as Planck.

Moreover, the BAOs capture only a part of information encoded in the shape of $P(k)$. One would miss another baryonic feature, the Silk damping scale, by only measuring BAOs. A more serious drawback is that one would miss the other prominent standard ruler, $k_{\mathrm{eq}}$, completely, by only measuring BAOs.

Nevertheless, there is one major advantage of using BAOs: the phases (not the amplitude) of BAOs are less sensitive to the distortion of the shape of $P(k)$ due to nonlinear matter clustering, nonlinear galaxy bias, or nonlinear redshift space distortion (Seo \& Eisenstein 2005; Eisenstein et al. 2007; Nishimichi et al. 2007; Smith et al. 2008; Angulo et al. 2008; Sanchez et al. 2008; Seo et al. 2008). As a result, many studies have focused on developing various ways to extract the distance information from BAOs.

Most of the previous work focused only on extracting the BAOs from the spherically averaged $P(k)$ (which gives $D_{A}^{2} / H$ ) (e.g., Percival et al. 2007). Yamamoto et al. (2005) have studied the monopole and quadrupole moments in the galaxy power spectrum and their implications for determinations of the dark energy equation of state parameter, $w$, and concluded that even in the worst case scenario (i.e., the absence of the BAOs feature on the observed power spectrum), galaxy survey can still provide useful limits on $w$ from a combination of the monopole and quadrupole power spectra. Recently, Padmanabhan \& White

\footnotetext{
4 The measured power spectrum in redshift space is a function of the wavenumber parallel to the line of sight, $k_{\|}$, and that perpendicular to the line of sight, $k_{\perp}$, i.e., $P=P\left(k_{\|}, k_{\perp}\right)$. The angular observables, $\theta_{\mathrm{eq}}$ and $\theta_{s}$, are measured from $k_{\perp}$, while the line-of-sight observables, $\delta z_{\mathrm{eq}}$ and $\delta z_{s}$, are measured from $k_{\|}$.
}

(2008) have explored an extraction of the quadrupole moment of the two-dimensional power spectrum, $P(k, \mu)$, which gives a different distance combination, $D_{A} H$.

In this paper, we shall develop a method for extracting $D_{A}$ and $H$ simultaneously from the two-dimensional BAOs. Since we do not use spherical averaging or truncate the Legendre expansion of BAOs at arbitrary orders, our method uses more information than most of the previous methods. To our knowledge, the full two-dimensional extraction of $D_{A}$ and $H$ from BAOs has been explored only by Wagner et al. (2008).

This paper is organized as follows. In Section 2, we give a brief account of the original one-dimensional "fit-andextract" (FITEX) method, which was developed by Koehler et al. (2007) for extracting BAOs from a spherically averaged one-dimensional $P(k)$. We then extend this method to the two-dimensional FITEX-2d method by including the full two-dimensional information without spherical averaging. In Section 3, we extract $D_{A}$ and $H$ from simulated noisy data using the FITEX-2d method, and show that the FITEX-2d yields unbiased estimates of $D_{A}$ and $H$. In Section, 4 we repeat the same analysis for a more realistic simulation, using the Millennium Simulation (Springel et al. 2005). In Section, 5 we propagate errors in $H(z)$ and $D_{A}(z)$ to those in the dark energy equation of state with the parametrization of $w(z)=w_{0}+w_{a} z /(1+z)$. We conclude in Section 6.

Throughout this paper we shall use the cosmological parameters given by $\Omega_{m}=0.277, \Omega_{\Lambda}=0.723, \Omega_{b}=0.0459$, $n_{s}=0.962$, and $h=0.702$ (Dunkley et al. 2009; Komatsu et al. 2009), which are the maximum likelihood values inferred from the WMAP five-year data (Hinshaw et al. 2009) combined with the current BAO data (Percival et al. 2007) and Type Ia supernova data (Kowalski et al. 2008).

\section{FITEX-2D: METHODOLOGY}

We develop a method for extracting $D_{A}$ and $H$ simultaneously from the two-dimensional BAOs without spherical averaging.

Our method builds upon the existing "fit-and-extract" (FITEX) method developed by Koehler et al. (2007) for extracting $D_{A}^{2} / H$ from a spherically averaged, one-dimensional $P(k)$. The FITEX method extracts BAOs by fitting and removing the nonoscillatory part of $P(k)$, which leaves only the oscillatory component, i.e., BAOs. Koehler et al. (2007) model the nonoscillatory, smooth part by the following functional form:

$$
P_{\text {smooth }}^{1 d}(k)=\left[\frac{A}{1+B k^{\delta}} e^{\left(k / k_{1}\right)^{\alpha}}\right]^{2} k^{n_{s}}
$$

where $n_{s}$ is the primordial tilt, while $A, B, \delta, k_{1}$, and $\alpha$ are free parameters. Koehler et al. (2007) have shown that this function is flexible enough to fit out the smooth part of the spherically averaged $P(k)$ measured from the Hubble Volume Simulation (Evrard et al. 2002). They have tested the FITEX method particularly for a large scale, $k<0.3 h \mathrm{Mpc}^{-1}$, at high redshifts, $1.9<z<3.8$, that are relevant to the Hobby-Eberly Dark Energy Experiment (HETDEX; Hill et al. 2004).

We make a simple extension of the one-dimensional FITEX method by including angular dependence. We model the twodimensional smooth power spectrum by

$$
\begin{aligned}
P_{\text {smooth }}^{2 d}(k, \mu)= & P_{\text {smooth }}^{1 d}(k)\left[1+g^{(2)}(k) P_{2}(\mu)\right. \\
& \left.+g^{(4)}(k) P_{4}(\mu)+g^{(6)}(k) P_{6}(\mu)\right],
\end{aligned}
$$

where $\mu$ is the cosine of the angle $\theta$ between $\mathbf{k}$ and the line of 
sight, i.e., $\mu=\cos \theta$ and $\tan \theta=k_{\perp} / k_{\|}$. Therefore, $\mu=0$ and $\mu=1$ for $k_{\|}=0$ and $k_{\perp}=0$, respectively.

Here, $P_{l}(\mu)$ is the Legendre polynomials:

$$
\begin{aligned}
& P_{2}(\mu)=\frac{1}{2}\left(3 \mu^{2}-1\right), \\
& P_{4}(\mu)=\frac{1}{8}\left(35 \mu^{4}-30 \mu^{2}+3\right), \\
& P_{6}(\mu)=\frac{1}{16}\left(231 \mu^{6}-315 \mu^{4}+105 \mu^{2}-5\right) .
\end{aligned}
$$

The odd multipoles must vanish by symmetry. One may include $l \geqslant 8$ if necessary, but we find it sufficient to include the terms only up to $l=6$.

Finally, $g^{(l)}(k)$ is given by the sixth-order polynomials with only even powers of $k$ :

$$
g^{(l)}(k)=a_{0}^{(l)}+a_{2}^{(l)} k^{2}+a_{4}^{(l)} k^{4}+a_{6}^{(l)} k^{6},
$$

where all of $a_{i}^{(l)}$ 's are varied simultaneously for each $l$. The odd powers must vanish because they are not analytic in $\mathbf{k}$ (Weinberg 2008). We include the terms only up to $k^{6}$, as we include the multipoles up to $l=6$. If, for instance, $l=8$ is included, then $k^{8}$ may also be included for consistency.

Aside from the primordial tilt, $n_{s}$, the FITEX-2d contains 17 free parameters (five for $P_{\text {smooth }}^{1 d}(k)$ plus $4 \times 3=12$ for the angular dependence). While it may sound like many, the number of data points available on the two-dimensional power spectrum is usually much larger, and thus our fit is well behaved.

It may be instructive to use the conventional model for the redshift space power spectrum to show what these parameters are supposed to capture. The leading order angular distortion is given by the so-called Kaiser effect, which arises from coherent converging velocity flow toward the linear overdensity region (Kaiser 1987). The linear Kaiser power spectrum is given by

$$
\begin{aligned}
P_{\text {kaiser }}^{\text {linear }}(k, \mu) & =b_{1}^{2}\left(1+2 \beta \mu^{2}+\beta^{2} \mu^{4}\right) P^{\text {linear }}(k) \\
& =b_{1}^{2}\left[\left(1+\frac{2}{3} \beta+\frac{1}{5} \beta^{2}\right)\right. \\
& \left.+\frac{4}{3} \beta\left(1+\frac{3}{7} \beta\right) P_{2}(\mu)+\frac{8}{35} \beta^{2} P_{4}(\mu)\right] P^{\text {linear }}(k),
\end{aligned}
$$

where $\beta \equiv f / b_{1}$ is a $k$-independent function that depends on the linear galaxy bias, $b_{1}$, and the cosmological parameters (mainly $\left.\Omega_{m}\right)$ via

$$
f \equiv \frac{d \ln D}{d \ln a}
$$

where $D$ is the growth factor of linear density fluctuations. We therefore find

$$
\begin{aligned}
& a_{0}^{(0)}=1 \\
& a_{0}^{(2)}=\frac{\frac{4}{3} \beta\left(1+\frac{3}{7} \beta\right)}{1+\frac{2}{3} \beta+\frac{1}{5} \beta^{2}}, \\
& a_{0}^{(4)}=\frac{\frac{8}{35} \beta^{2}}{1+\frac{2}{3} \beta+\frac{1}{5} \beta^{2}},
\end{aligned}
$$

and the other terms are zero.

Another example is the so-called finger-of-God (FoG) effect, which arises from random motion within virialized halos. When

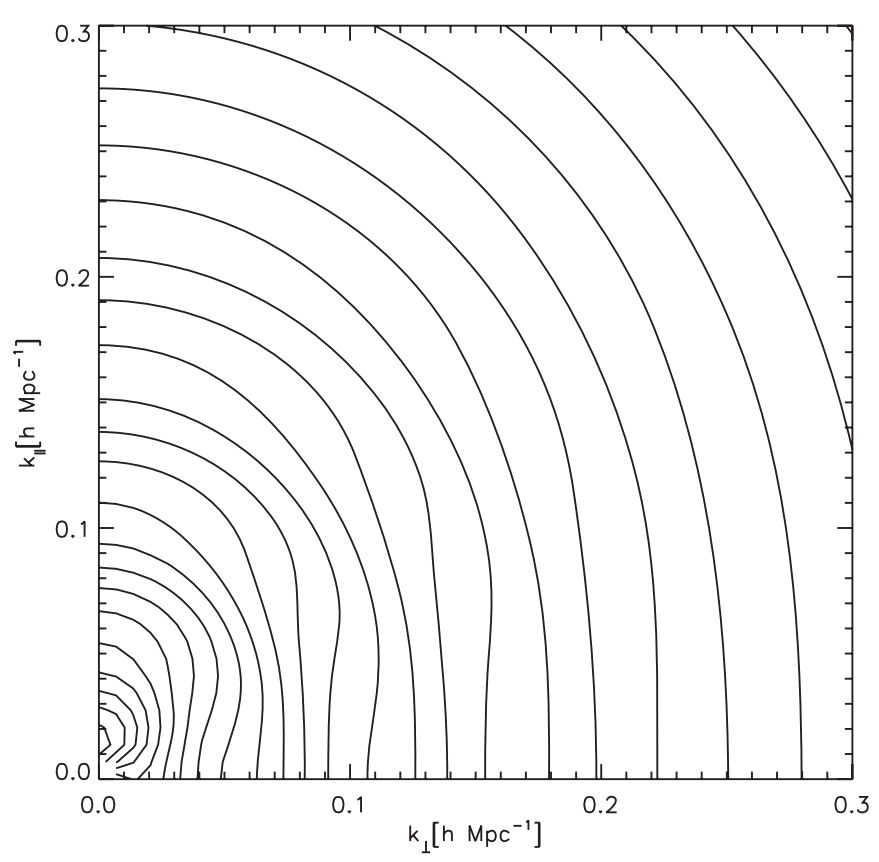

Figure 1. Illustration of the FITEX-2d method. This figure shows an anisotropic nonlinear galaxy power spectrum before we apply FITEX-2d. The contours show $\ln \left[P\left(k_{\|}, k_{\perp}\right)\right]$ at $z=2$, where we have computed $P\left(k_{\|}, k_{\perp}\right)$ from Equation (21). Anisotropic distribution of power due to redshift space distortion is apparent.

the distribution of the pairwise peculiar velocity within a halo is given by an exponential distribution with the velocity dispersion $\sigma_{v}^{2}$ (Peebles 1976; Davis \& Peebles 1983), one finds (Ballinger et al. 1996)

$$
P_{F o G}(k, \mu)=\frac{P_{\text {kaiser }}^{\text {linear }}(k, \mu)}{1+f^{2} k^{2} \mu^{2} \sigma_{v}^{2}} .
$$

While the FoG yields many terms when expanded into the Legendre polynomials, it is still a good approximation to truncate the expansion at $l=6$ if $k$ is sufficiently smaller than $1 / \sigma_{v}$. Note that the FoG effect yields terms in the form of powers of $(k \mu)^{2}$; thus, it makes sense to use the same number for the maximum power of $k$ (see Equation 14) and the maximum multipole (see Equation 10) of the FITEX-2d fitting function.

In general, neither of these two expressions are adequate. The linear Kaiser formula is valid only on very large scales, while the exponential FoG formula is valid only on very small scales. At the intermediate scales we find more complicated expressions from, e.g., the third-order perturbation theory (Heavens et al. 1998). To account for these complications we have included $k$-dependent coefficients for the Legendre polynomials.

In Figures 1 and 2 we show the performance of $P_{\text {smooth }}^{2 d}(k, \mu)$. In Figure 1 we show a simple analytical $\operatorname{model}^{5}$ for the nonlinear galaxy power spectrum in redshift space given by

$$
\begin{aligned}
P_{g}(k, \mu)= & b_{1}^{2}\left[P_{\delta \delta}(k)+2 \beta \mu^{2} P_{\delta \theta}(k)+\beta^{2} \mu^{4} P_{\theta \theta}(k)\right] \\
& \times \frac{1}{1+f^{2} k^{2} \mu^{2} \tilde{\sigma}_{v}^{2}},
\end{aligned}
$$

where $P_{\delta \delta}(k), P_{\delta \theta}(k)$, and $P_{\theta \theta}(k)$ are the density-density, density-velocity, and velocity-velocity power spectra computed from the third-order perturbation theory, and they are

5 This model is admittedly too simple to be realistic. We shall test the FITEX-2d method in a more realistic setting using the Millennium Simulation in Section 4. 


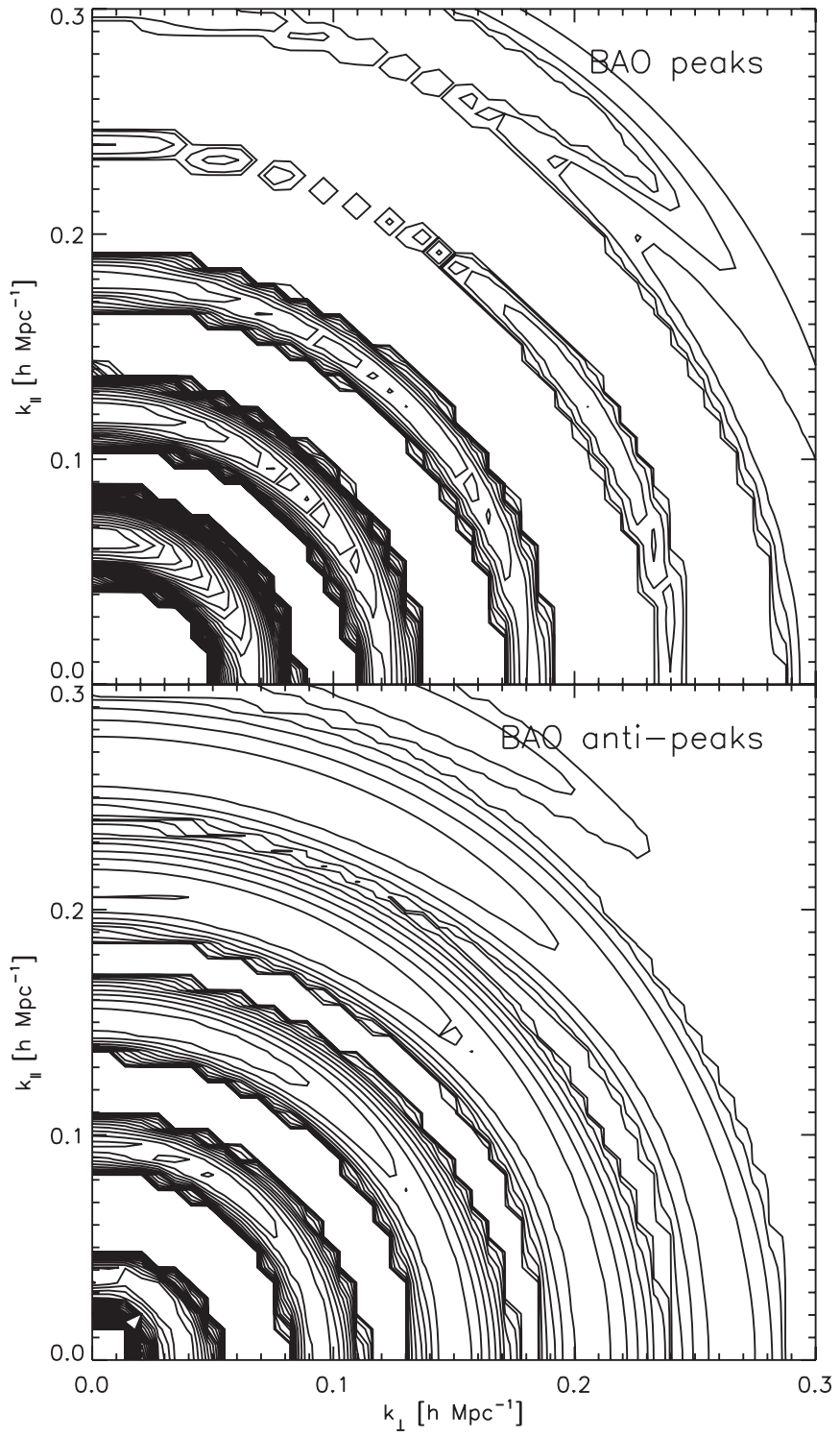

Figure 2. Illustration of the FITEX-2d method. This figure shows the power spectrum shown in Figure 1 minus the best-fitting two-dimensional smooth spectrum, $P_{\text {smooth }}^{2 d}\left(k_{\|}, k_{\perp}\right)$, given by Equation (10). The structure of BAOs, i.e., the oscillatory feature, is now apparent. The FITEX-2d method recovers the isotropic distribution of the BAO phases successfully, which makes it possible to use the distribution of the phases for measuring $D_{A}$ and $H$ simultaneously. Top: positive BAO peaks; bottom: negative BAO peaks (troughs).

given by Equations (63), (64), and (65) in Scoccimarro (2004), respectively. This form is similar to Equation (71) of Scoccimarro (2004), but we have replaced $\exp \left(-f^{2} k^{2} \mu^{2} \sigma_{v}^{2}\right)$ and $f$ in his formula by $1 /\left(1+f^{2} k^{2} \mu^{2} \tilde{\sigma}_{v}^{2}\right)$ and $\beta$, respectively, where $\tilde{\sigma}_{v}^{2} \equiv 0.6 \sigma_{v}^{2}$ is the one-dimensional peculiar velocity dispersion with an empirical fudge factor of 0.6 calibrated off our simulations presented in Jeong \& Komatsu (2006). Here, $\sigma_{v}^{2}$ is given by

$$
\sigma_{v}^{2} \equiv \frac{1}{3} \int \frac{d^{3} k}{(2 \pi)^{3}} \frac{P^{\text {linear }}(k)}{k^{2}}=\frac{1}{3} \int \frac{d k}{2 \pi^{2}} P^{\text {linear }}(k) .
$$

We chose $z=2$ and $b_{1}=2.5$. The contour of power spectrum is anisotropic in Figure 1 due to the redshift space distortion; however, we recover isotropy after subtracting the best-fitting $P_{\text {smooth }}^{2 d}(k, \mu)$ from the anisotropic data (see Figure 2). We see that the BAOs have been extracted successfully, with isotropy of the oscillation phases recovered well.

\section{EXTRACTION OF $D_{A}$ AND $H$ FROM NOISY DATA: FITEX-2D VERSUS FULL MODELING}

In Section 3.1, we show how well we can estimate $D_{A}$ and $H$ from the two-dimensional BAOs extracted from noisy data using the FITEX-2d method. In Section 3.2, we compare the BAO results to the accuracy one would obtain from the full modeling of $P(k, \mu)$, including the overall shape. In other words, for the former (BAOs) we only use $\theta_{s}$ and $\delta z_{s}$ for measuring $D_{A}$ and $H$, while for the latter (full modeling) we can use $\theta_{s}, \delta z_{s}, \theta_{\text {eq }}$, $\delta z_{\text {eq }}$, as well as the Silk damping scale for measuring $D_{A}$ and $H$, provided that nonlinear effects (nonlinear matter clustering, nonlinear redshift space distortion, and nonlinear bias) are under control.

Note that the treatment of nonlinear effects in this section is too simple to be realistic. For a more realistic treatment, we shall use the galaxy power spectrum from the Millennium Simulation (Springel et al. 2005) in Section 4.

\subsection{FITEX-2d}

To estimate errors in $D_{A}$ and $H$ from the FITEX-2d method, we use simple Monte Carlo simulations.

For the underlying spectrum we use the same data as shown in Figure 1, which includes a simplified modeling of nonlinear matter clustering and nonlinear redshift space distortion as given by Equation (21). As for the galaxy bias, we use a linear bias with $b_{1}=2.5$.

Once the underlying spectrum is specified, it is straightforward to compute the errors in $P_{g}\left(k_{\|}, k_{\perp}\right), \sigma_{P_{g}}$, provided that the distribution of $P_{g}\left(k_{\|}, k_{\perp}\right)$ is a Gaussian. We use the standard formula that includes sampling variance as well as shot noise (see, e.g., Jeong \& Komatsu 2009)

$$
\frac{\sigma_{P_{g}}\left(k_{\|}, k_{\perp}\right)}{P_{g}\left(k_{\|}, k_{\perp}\right)}=2 \pi \sqrt{\frac{1}{V_{\text {survey }} k_{\perp} \Delta k_{\perp} \Delta k_{\|}}} \frac{1+n_{g} P_{g}\left(k_{\|}, k_{\perp}\right)}{n_{g} P_{g}\left(k_{\|}, k_{\perp}\right)},
$$

where $n_{g}$ is the number density of galaxies, $V_{\text {survey }}$ is the survey volume, $\Delta k_{\perp}$ and $\Delta k_{\|}$are the fundamental wavenumbers, i.e., the resolution in $k_{\perp}$ and $k_{\|}$. We take these to be $\Delta k_{\|}=\Delta k_{\perp}=$ $(2 \pi) / V_{\text {survey. }}^{1 / 3}$.

We use $\sigma_{P_{g}}$ from Equation (23) to calculate the rms error in $P_{g}\left(k_{\|}, k_{\perp}\right)$, and generate 1000 Monte Carlo realizations. We then apply the FITEX-2d method to remove the smooth component from each realization to extract BAOs. For each realization, we measure $D_{A}$ and $H$ simultaneously by fitting the phases of extracted two-dimensional BAOs to those of the reference BAOs extracted from either (1) the linear power spectrum, or (2) the nonlinear power spectrum given by Equation (21), with known $D_{A \text {,ref }}$ and $H_{\text {ref }}$. (Later we find that using the linear spectrum as the reference BAO yields the biased estimates of $D_{A \text {,ref }}$ and $H_{\text {ref.) }}$ We use a simplex downhill method for $\chi^{2}$-minimization in the two-dimensional parameter space. The number of free parameters for this analysis is two, i.e., $D_{A}$ and $H$, and we do not include the amplitude in the fit. We have checked that including the amplitude does not change the results very much, as the amplitude and the phases of BAOs are nearly uncorrelated (see Appendix A.3.1 for more details). This is true in both real and redshift space. When we apply FITEX-2d to the simulated data, we perform a fit out to $k_{\max }=0.40 \mathrm{~h} \mathrm{Mpc}^{-1}$. 


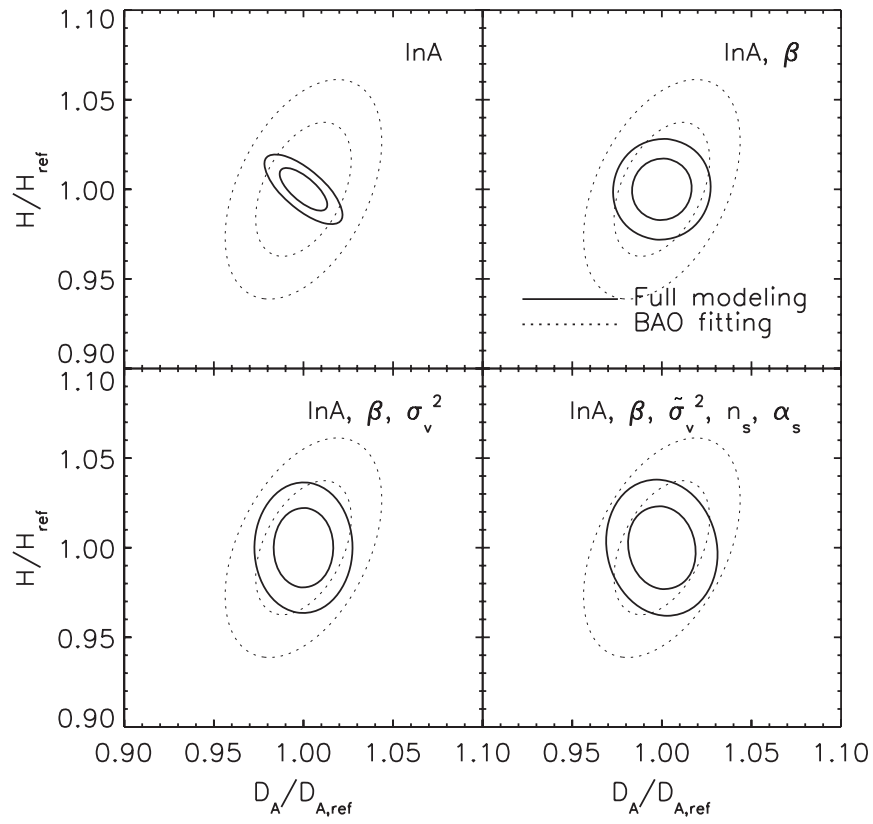

Figure 3. Accuracy of $D_{A}$ and $H$ extracted from BAOs with the FITEX-2d method applied to simulated Monte Carlo realizations that approximate the HETDEX survey (the larger, dotted contours; see Section 3.1). The best-fitting values of $D_{A}$ and $H$ agree with the true values; thus, the FITEX-2d method yields unbiased estimates of $D_{A}$ and $H$. The solid contours show $D_{A}$ and $H$ from the full modeling, including the overall shape of the power spectrum, with various parameters marginalized over. (Note that the BAO-only contours are unaffected by the marginalization.) For this we have used the Fisher matrix forecast (see Section 3.2). The inner and outer ellipses show $68 \%$ and 95\% C.L., respectively. (Top left) The full modeling Fisher matrix is marginalized over the overall amplitude, $\ln A$, (top right) marginalized over $\ln A$ and the linear redshift distortion parameter, $\beta$, (bottom left) marginalized over $\ln A, \beta$, and the velocity dispersion in the FoG factor, $\tilde{\sigma}_{v}^{2}$, (bottom right) marginalized over $\ln A, \beta, \tilde{\sigma}_{v}^{2}$, and the shape of the initial power spectrum, $n_{s}$ and $\alpha_{s}$.

We choose the survey parameters, $V_{\text {survey }}, z$, and $n_{g}$, such that they roughly match those expected for the HETDEX (Hill et al. 2004): $N_{g}=0.755 \times 10^{6}$, and $1.9 \leqslant z \leqslant 3.5$ with the sky coverage of $420 \mathrm{deg}^{2}$, which yields $V_{\text {survey }} \simeq 3.0 h^{-3} \mathrm{Gpc}^{3} .6$

We find that, when the phases extracted by FITEX-2d are compared with the reference BAOs extracted from the linear power spectrum, the best-fitting values of $D_{A}$ and $H$ averaged over 1000 simulations disagree with the underlying, "true" values by $0.05 \%$ and $0.63 \%$ for $D_{A}$ and $H$, respectively, due to the phase shift of BAOs caused by nonlinearities (including nonlinear redshift space distortion). This result extends the previous study by Nishimichi et al. (2007), who studied a spherically averaged one-dimensional power spectrum and found that the bias was less than $1 \%$ in $\left(D_{A}^{2} H^{-1}\right)^{1 / 3}$.

On the other hand, when the phases are compared with the reference BAOs extracted from the nonlinear power spectrum (Equation 21), the best-fitting values of $D_{A}$ and $H$ agree with the true values to well within the Monte Carlo sampling error; thus, we confirm that the FITEX-2d method yields unbiased estimates of $D_{A}$ and $H$.

In Figure 3 we show the projected error ellipses on $D_{A}$ and $H$ from the BAOs extracted with the FITEX-2d (larger, dotted contours; same in all four panels). We find $1.8 \%$ and $2.5 \%$ errors on $D_{A}$ and $H$, respectively, with the cross-correlation coefficient of $r=0.44$, from the Monte Carlo simulations. For the same

\footnotetext{
6 The HETDEX is expected to detect 0.755 million Lyman- $\alpha$ emitting galaxies between $1.9 \leqslant z \leqslant 3.5$ over $420 \mathrm{deg}^{2}$ in three years of observations on the Hobby-Eberly Telescope.
}

survey parameters, the BAO Fisher matrix proposed by Seo \& Eisenstein (2007) yields $1.5 \%$ and $2.5 \%$ errors on $D_{A}$ and $H$, respectively, with $r=0.41$. Therefore, we conclude that the FITEX-2d method yields the results that nearly saturate the Fisher matrix bound, i.e., it is nearly an optimal method in a sense that it can yield the smallest errorbars one can obtain with the BAO-only analysis.

\subsection{Full Modeling}

To calculate the errors in $D_{A}$ and $H$ expected from the full modeling of the two-dimensional galaxy power spectrum, $P_{g}(k, \mu)$, we use the Fisher matrix given by (see, e.g., Eisenstein et al. 1999; Seo \& Eisenstein 2003)

$$
F_{i j}=\int_{0}^{k_{\max }} \frac{4 \pi k^{2} d k}{(2 \pi)^{3}} \int_{0}^{1} d \mu \frac{\partial \ln P_{g}(k, \mu)}{\partial \theta_{i}} \frac{\partial \ln P_{g}(k, \mu)}{\partial \theta_{j}} w(k, \mu),
$$

where $\theta_{i}=\left(\ln D_{A}, \ln H, \ln A, \beta, \tilde{\sigma}_{v}^{2}, n_{s}, \alpha_{s}\right)$ for $i=1,2, \ldots, 7$, respectively, $k_{\max }=0.40 \mathrm{~h} \mathrm{Mpc}^{-1}$, where $A$ is the overall amplitude of the power spectrum, $\beta$ is the linear redshift distortion parameter, $\tilde{\sigma}_{v}^{2}$ is the calibrated one-dimensional velocity dispersion (see Equation 21), and $n_{s}$ and $\alpha_{s}$ describe the shape of the initial (primordial) power spectrum:

$$
P_{i n i}(k) \propto k^{n_{s}+\frac{1}{2} \alpha_{s} \ln \left(k / k_{\text {pivot }}\right)} .
$$

Here, the weight function, $w(k, \mu)$, is one half of the so-called "effective volume,"

$$
w(k, \mu) \equiv \frac{1}{2}\left[\frac{n_{g} P_{g}(k, \mu)}{1+n_{g} P_{g}(k, \mu)}\right] V_{\text {survey }} \equiv \frac{1}{2} V_{\text {eff }}(k, \mu) .
$$

The effective volume is equal to the actual survey volume, $V_{\text {survey }}$, in the sampling variance dominated regime, $P_{g}(k, \mu) \gg$ $1 / n_{g}$, whereas it is small in the shot-noise dominated regime, $P_{g}(k, \mu) \ll 1 / n_{g}$. In Figure 4 we show $n_{g} P_{g}(k, \mu)$ for $N_{g}=$ $0.755 \times 10^{6}$ and $b_{1}=2.5$ as a function of $z$. The factor of $1 / 2$ accounts for symmetry in $\mathbf{k} \rightarrow-\mathbf{k}$. The derivatives of ln $P_{g}(k, \mu)$ with respect to $\theta_{i}$ are calculated and given in the appendix A.2.

Unlike for BAOs, which are insensitive to the parameters that affect the overall shape, for the full modeling we need to make sure that we take into account potential degeneracy between $D_{A}$ and $H$ and any other parameters that affect the overall shape. In this paper, we include $\ln A, \beta, \tilde{\sigma}_{v}{ }^{2}, n_{s}$, and $\alpha_{s}$. (We shall comment on the effects of nonlinear bias in Section 3.3.)

We study the effects of marginalization over various parameter combinations by taking the submatrix, $\bar{F}_{i j}$, of the full $7 \times 7$ matrix with the index, $i$, of $\theta_{i}$ running from 1 to 7 , such that the submatrix includes the matrix components of desired parameters to be marginalized. In other words, the parameters that are not included in the submatrix are fixed and not marginalized over.

Then, we compute the marginalized errors in $\ln D_{A}$ and $\ln H$ as

$$
\begin{gathered}
\sigma_{\ln D_{A}}=\sqrt{\left(\bar{F}^{-1}\right)_{11}}, \\
\sigma_{\ln H}=\sqrt{\left(\bar{F}^{-1}\right)_{22}} .
\end{gathered}
$$

To simplify the analysis, we fix all the other cosmological parameters, such as $f(z), \Omega_{b} h^{2}$, etc. These cosmological parameters will be determined by the future CMB mission, Planck, 
Table 1

Fisher Matrix Forecast for the Full Power Spectrum Analysis with Various Choices of Marginalization

\begin{tabular}{|c|c|c|c|c|c|c|c|}
\hline & None & $\ln A$ & $\beta$ & $\tilde{\sigma}_{v}^{2}$ & $n_{s}$ & $\alpha_{s}$ & $\ln A, \beta$ \\
\hline$\Delta \ln D_{A}(\%)$ & 0.279 & 0.876 & 0.318 & 0.282 & 0.479 & 0.416 & 1.100 \\
\hline$\Delta \ln H(\%)$ & 0.433 & 0.784 & 1.118 & 0.794 & 0.506 & 0.535 & 1.127 \\
\hline$r_{D_{A}, H}$ & 0.379 & -0.723 & -0.312 & 0.080 & -0.232 & -0.230 & 0.033 \\
\hline \multirow[t]{2}{*}{$\Delta \ln R(\%)$} & 0.187 & 0.761 & 0.318 & 0.259 & 0.386 & 0.363 & 0.774 \\
\hline & $\beta, \tilde{\sigma}_{v}^{2}$ & $\ln A, \tilde{\sigma}_{v}^{2}$ & $\ln A, \beta, \tilde{\sigma}_{v}^{2}$ & $\ln A, n_{s}, \alpha_{s}$ & $\begin{array}{c}\ln A, \beta, n_{s} \\
\alpha_{s}\end{array}$ & $\begin{array}{c}\ln A, \beta, \tilde{\sigma}_{v}^{2}, \\
n_{s}, \alpha_{s}\end{array}$ & \\
\hline$\Delta \ln D_{A}(\%)$ & 0.327 & 0.891 & 1.101 & 1.088 & 1.232 & 1.250 & \\
\hline$\Delta \ln H(\%)$ & 1.448 & 1.096 & 1.459 & 0.982 & 1.355 & 1.521 & \\
\hline$r_{D_{A}, H}$ & -0.386 & -0.635 & 0.001 & -0.822 & -0.204 & -0.103 & \\
\hline$\Delta \ln R(\%)$ & 0.322 & 0.868 & 0.878 & 0.973 & 1.000 & 1.013 & \\
\hline
\end{tabular}

Notes. The fractional errors in $D_{A}$ and $H$, and their cross-correlation coefficients, $r_{D_{A}, H}$, and the fractional errors in the combined one-dimensional distance scale, $R$ (Equation 29), marginalized over several combinations of parameters: $\ln A, \beta, \tilde{\sigma}_{v}^{2}, \alpha_{s}$ and $n_{s}$. The cosmological parameters are taken from Table 1 of the Komatsu et al. (2009; "WMAP+BAO+SN ML"). The survey parameters approximate those of HETDEX: the survey area and target redshift are $420 \mathrm{deg}^{2}$ and $1.9<z<3.5$, respectively, the number of galaxies is $N_{g}=0.755 \times 10^{6}$, and the bias is assumed to be linear with $b_{1}=2.5$.

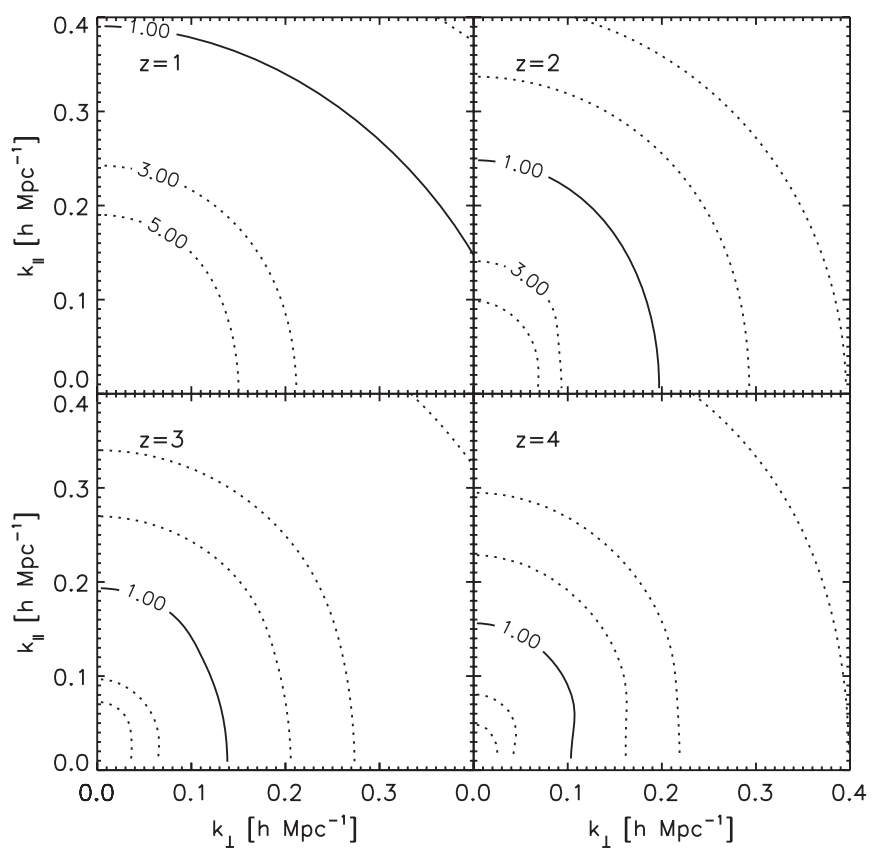

Figure 4. Galaxy power spectrum times the number density of galaxies, $n_{g} P_{g}(k, \mu)$, where the number of the galaxies is fixed for each redshift bin to $N_{g}=0.755 \times 10^{6}$, and $P_{g}(k, \mu)$ is computed from Equation (21) with $b_{1}=2.5$. The shot noise dominates the error budget when $n_{g} P_{g}(k, \mu)<1$. Contour values are $[0.1,0.3,0.5,1.0,3.0,5.0]$. (Top left) $z=1$, (top right) $z=2$, (bottom left) $z=3$, (bottom right) $z=4$.

accurately, and therefore it is a good approximation to simply fix them, and vary only $\ln D_{A}, \ln H, A, \beta, \tilde{\sigma}_{v}^{2}, n_{s}$ and $\alpha_{s}$. The fiducial value for the bias is set to $b_{1}=2.5$ and $f=d \ln D / d \ln a$ is computed from the fiducial cosmological model. ${ }^{7}$ We expect that the analysis of the bispectrum (Fourier transform of the three-point function) will give a precise determination of $b_{1}$ (as well as nonlinear bias parameters such as $b_{2}$; Sefusatti \& Komatsu 2007), and therefore it is also a good approximation to simply fix it. However, we also explore a more conservative

\footnotetext{
7 One might also wish to marginalize over $f$ for the following reason: while $f$ can be calculated from the cosmological parameters assuming the validity of general relativity, one might choose to let $f$ free and use it for testing the validity of general relativity. In this paper, we chose to assume the validity of general relativity, but one can extend our analysis to let $f$ free in a straightforward manner.
}

case where we do not know what $b_{1}$ is, i.e., we marginalize over the overall amplitude as well as $\beta$. In the future work we also plan to investigate the effect of marginalization over $b_{2}$, using a joint analysis of the power spectrum and bispectrum. Therefore, our calculation presented here will provide the lower limit to the errors in $\ln D_{A}$ and $\ln H$ expected from the full modeling of the power spectrum measured in a survey like HETDEX. We use the same survey parameters that we have used in Section 3.1, and we integrate Equation (24) up to $k_{\max }=0.40 \mathrm{~h} \mathrm{Mpc}^{-1}$.

In Figure 3, we show the resulting error ellipses from the full modeling, in the smaller, solid contours, with four choices of marginalization (we present the results from more choices of marginalization in Table 1). First, for all choices of marginalization we find that the sizes of the errors in both $D_{A}$ and $H$ are substantially smaller than those from the BAOonly analysis with the FITEX-2d. For example, determinations of both $D_{A}$ and $H$ are improved by more than a factor of 2 in the case of the amplitude marginalization. This is expected, as we are able to use more information encoded in the power spectrum; namely, the Hubble horizon at the matter-radiation equality epoch and the Silk damping scale. Second, $D_{A}$ and $H$ are anti-correlated for the amplitude marginalization, with the cross-correlation coefficient of $r=-0.72$ (see the top-left panel of Figure 3), as opposed to a positive correlation seen in the BAO-only analysis. This is due to the marginalization over the overall amplitude: if we fixed the overall normalization, then we would still find a positive correlation between $D_{A}$ and $H$ with $r=0.38$.

The origin of the negative correlation is the so-called AlcockPaczynski (AP) test (Alcock \& Paczynski 1979): when the redshift space distortion is known perfectly well, the departure of the power spectrum in redshift space from isotropy, i.e., dependence of $P(k, \mu)$ on $\mu^{2}$, can be used to determine $D_{A H}$, resulting in $r=-1$ for a power-law power spectrum. The contributions from departures of $P(k)$ from a pure power-law, i.e., the existence of "standard rulers," such as BAOs, the Hubble horizon at the matter-radiation equality and the Silk damping scale, make $r$ bigger than -1 (see Appendix A.3 for more details). When $\ln A$ and $\beta$ are marginalized over simultaneously, the correlation between $D_{A}$ and $H$ nearly disappears: the AP test no longer works when we marginalize over the linear redshift space distortion. We find $r=0.033$ (see the top right panel of Figure 3) for this case. 
When $\ln A$ is marginalized over while the other parameters $\left(\beta, \tilde{\sigma}_{v}^{2}, n_{s}\right.$, and $\left.\alpha_{s}\right)$ are held fixed, we find $0.88 \%$ and $0.78 \%$ errors on $D_{A}$ and $H$, respectively, with $r=-0.72$. The more parameters we marginalize over, the greater the crosscorrelation coefficient between $D_{A}$ and $H$ as well as the errors on $D_{A}$ and $H$ become. Note that the increase in the errors does not necessarily imply the decrease in the statistical power in constraining dark energy properties: since the cross-correlation coefficient is also reduced, the error in the combined onedimensional distance scale, $R$, is much less affected by the marginalization (see Table 1). The error in $\ln R$ has been computed as (Seo \& Eisenstein 2007)

$$
\sigma_{\ln R}^{2}=\frac{\sigma_{\ln D_{A}}^{2}\left(1-r^{2}\right)}{1+2 r \sigma_{\ln D_{A}} / \sigma_{\ln H}+\sigma_{\ln D_{A}}^{2} / \sigma_{\ln H}^{2}} .
$$

Finally, the errors in $D_{A}, H$, and $R$ for various choices of marginalization are $\left(\sigma_{\ln D_{A}}, \sigma_{\ln H}, \sigma_{\ln R}\right)=(0.88 \%, 0.78 \%$, $0.76 \%),(1.10 \%, 1.13 \%, 0.77 \%),(1.10 \%, 1.46 \%, 0.88 \%)$, and $(1.25 \%, 1.52 \%, 1.01 \%)$ for the marginalization over $\ln A$, $\ln A$ and $\beta, \ln A, \beta$ and $\tilde{\sigma}_{v}^{2}$, and $\ln A, \beta, \tilde{\sigma}_{v}^{2}, n_{s}$ and $\alpha_{s}$, respectively (see Table 1 for a more comprehensive list). This result should be compared with that from the BAO-only analysis: $\left(\sigma_{\ln D_{A}}, \sigma_{\ln H}, \sigma_{\ln R}\right)=(1.76 \%, 2.47 \%, 1.08 \%)$. It is clear that the full analysis, even with a generous set of marginalization choices, beats the BAO-only analysis with a significant gain in the distance determination accuracies.

\subsection{Caveat for the Full Modeling}

Our analysis presented in Section 3.2 is too simplistic and optimistic, as it ignores any systematic errors due to our lack of understanding of the effects of various nonlinearities in the power spectrum.

Among the three major nonlinearities, nonlinear matter clustering is under control, at least for high redshifts, i.e., $z \gtrsim 2$, as one can model nonlinear evolution of matter fluctuations almost exactly by the third-order perturbation theory (Jeong \& Komatsu 2006). While the nominal third-order perturbation theory breaks down at lower redshifts, $z \sim 1$, there have been a number of studies aiming at improving upon our ability to compute $P(k)$ at $z \sim 1$ or even lower redshifts (Crocce \& Scoccimarro 2008; Matarrese \& Pietroni 2007; Taruya \& Hiramatsu 2008; Valageas 2007; Matsubara 2008; McDonald 2007). Therefore, it is quite possible that the nonlinear matter clustering will be fully under control in the near future, at least for the scales that are relevant to the BAO scales, i.e., $k \lesssim 0.40 h \mathrm{Mpc}^{-1}$.

In a separate paper (Jeong \& Komatsu 2009), we show that nonlinear galaxy biasing is also under control in the weakly nonlinear regime. One can use the perturbation theory approach combined with the local bias assumption (Fry \& Gaztanaga 1993; McDonald 2006) to model the galaxy power spectrum with nonlinear bias.

The most problematic one is the nonlinear redshift space distortion. Our understanding of nonlinear redshift space distortion, especially the FoG effect, is limited (Scoccimarro 2004). Therefore, whether one can achieve the accuracy of $D_{A}$ and $H$ ( $H$ in particular) reported in Figure 3 depends crucially on our ability to correct for the FoG effect. This is work in progress. Note that the marginalization over $\tilde{\sigma}_{v}^{2}$ should capture some of the increase in the errors in distance scales due to our ignorance of FoG.

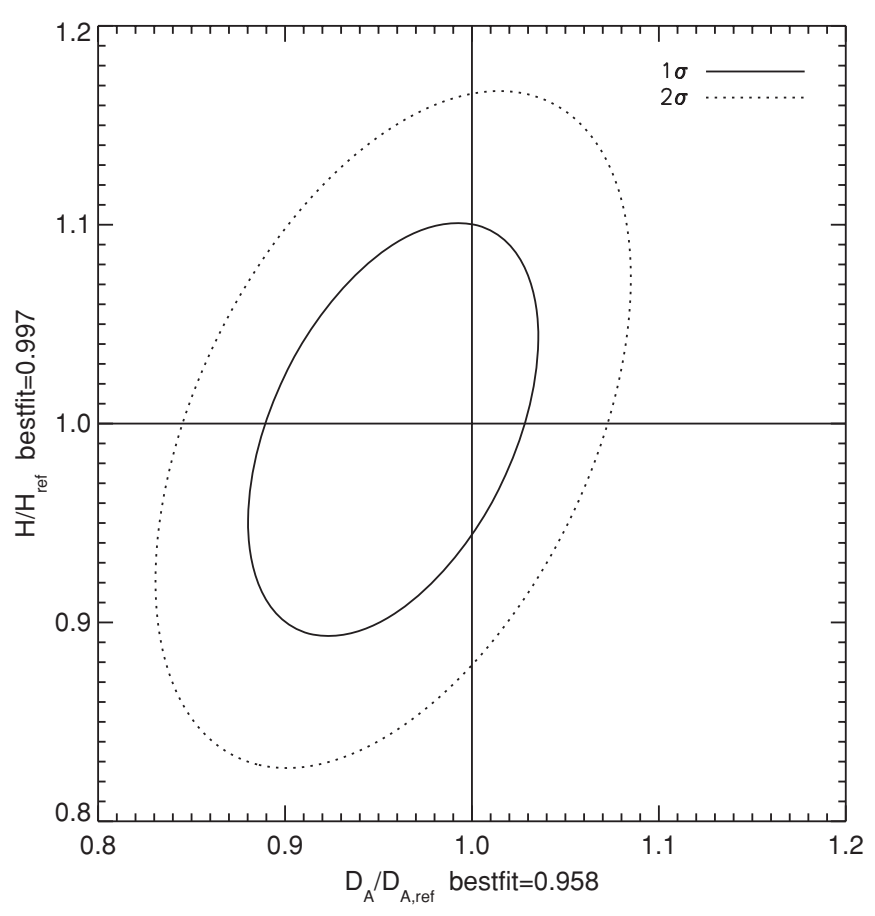

Figure 5. Accuracy of $D_{A}$ and $H$ extracted from BAOs with the FITEX-2d method applied to the Millennium Galaxy Simulation in redshift space at $z=3$ (Springel et al. 2005; Bower et al. 2006; Benson et al. 2003; Cole et al. 2000). The best-fitting values of $D_{A}$ and $H$ agree with the true values to within statistical errors of the Millennium Simulation; thus, the FITEX-2d method also yields unbiased estimates of $D_{A}$ and $H$ for the Millennium Simulation. The solid and dotted lines show $68 \%$ and $95 \%$ C.L., respectively.

\section{EXTRACTION OF $D_{A}$ AND $H$ FROM THE MILLENNIUM SIMULATION}

How realistic is our result for the determinations of $D_{A}$ and $H$ from the BAO phases using the FITEX-2d method? Since our Monte Carlo simulations used in Section 3.1 are too simple, in this section we test the FITEX-2d method further by using the Millennium Simulation (Springel et al. 2005).

We use the Millennium Galaxy catalogue, generated by the semi-analytical galaxy formation code (Bower et al. 2006; Benson et al. 2003; Cole et al. 2000). We have measured the twodimensional power spectrum of galaxies in redshift space from the Millennium Simulation, and applied the FITEX-2d method to remove the smooth component. We then find the best-fitting $D_{A}$ and $H$ from the BAO phases extracted from the FITEX-2d. Again, we use the data up to $k_{\max }=0.40 h \mathrm{Mpc}^{-1}$.

In Figure 5 we show the result. The best-fitting value that we find from the Millennium Simulation corresponds to one point at the center of the contours. We find the errors from the Monte Carlo simulations that we described in Section 3.1 with the survey parameters replaced by those of the Millennium Simulation: $V_{\text {survey }}=\left(0.5 h^{-1} \mathrm{Gpc}\right)^{3}, n_{g}=0.138 h^{3} \mathrm{Mpc}^{-3}$, and $z=3.06$. (There are 17,238,935 galaxies in the Millennium Simulation at $z=3.06$.) For the theoretical power spectrum that we use for generating Monte Carlo simulations, we use the best-fitting power spectrum for the galaxy catalogue of the Millennium Simulation found in Jeong \& Komatsu (2009).

Since the volume of the Millennium Simulation is $\sim 24$ times as small as that would be surveyed by HETDEX, the uncertainties in $D_{A}$ and $H$ are larger for the Millennium Simulation. (Compare Figure 5 with the larger contours of Figure 3.) We find $5.1 \%$ and $6.8 \%$ errors on $D_{A}$ and $H$, 


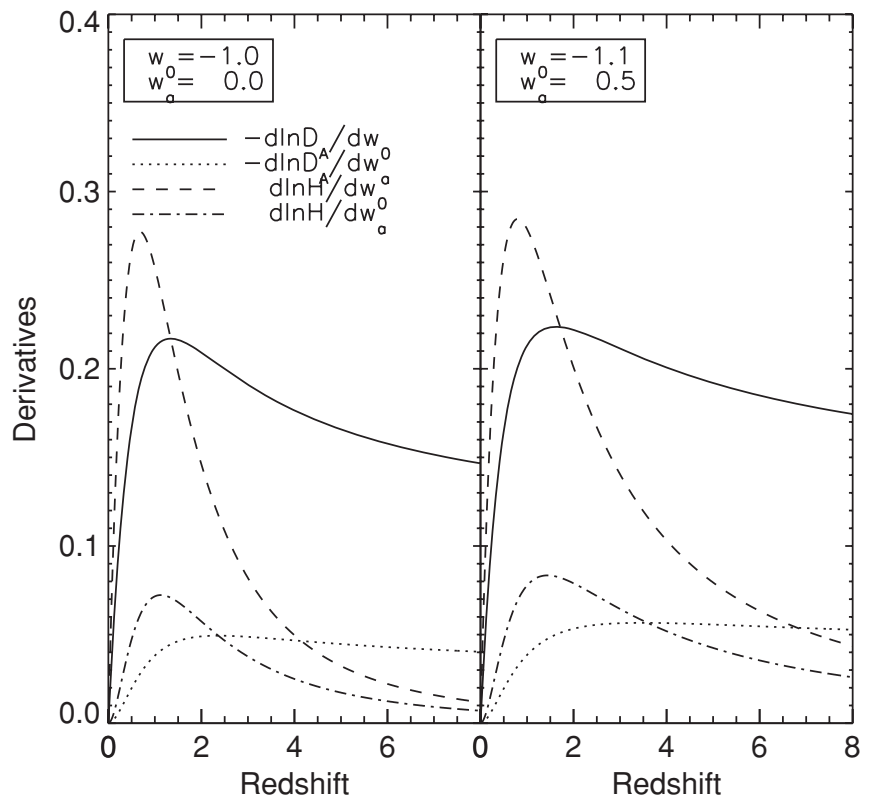

Figure 6. Partial derivatives of $\ln D_{A}$ and $\ln H$ with respect to the dark energy equation of state parameters, $w_{0}$ and $w_{a}$, as a function of $z$ for two different cosmological models. (Left) $\left(w_{0}, w_{a}\right)=(-1.0,0.0)$. (Right) $\left(w_{0}, w_{a}\right)=(-1.1,0.5)$.

respectively, with the cross-correlation coefficient of $r=0.43$, from the Monte Carlo simulations. These errors are larger than those from HETDEX Monte-Carlo simulation by a factor of 2 (rather than $\sqrt{24} \sim 5$ ) as the shot noise on the power spectrum of the Millennium Simulation is much smaller than that of HETDEX simulation.

The best-fitting values of $D_{A}$ and $H$ are well within the $68 \%$ confidence level (C.L.) region, which indicates that the FITEX$2 \mathrm{~d}$ is able to yield unbiased estimates of the BAO phases from the Millennium Simulation.

These results indicate that the FITEX-2d method that we have developed in this paper can be used for extracting the BAOs and measuring $D_{A}$ and $H$ safely from the real data. It would be interesting to apply the FITEX-2d method to the twodimensional power spectrum measured from the SDSS LRG samples (Okumura et al. 2008), and extract $D_{A}$ and $H$ from them.

\section{ERROR PROPAGATION TO THE DARK ENERGY EQUATION OF STATE}

In Sections 3 and 4, we have estimated errors in $D_{A}$ and $H$ from two different approaches, i.e., the BAO fitting using the FITEX-2d method and the full modeling. In this section, we propagate errors in $D_{A}$ and $H$ to those in the dark energy equation of state parameters. We parametrize $w(z)$ using the linear model, $w(z)=w_{0}+w_{a} z /(1+z)$ (Linder 2003; Chevallier \& Polarski 2001). by

We propagate the errors in $D_{A}$ and $H$ to those in $w_{0}$ and $w_{a}$

$$
\tilde{F}_{\alpha \beta}=\sum_{i j} \frac{\partial p_{i}}{\partial q_{\alpha}} \frac{\partial p_{j}}{\partial q_{\beta}} F_{i j}
$$

where $\tilde{F}_{\alpha \beta}$ is the Fisher matrix for the dark energy parameters, $F_{i j}$ is the Fisher matrix for $D_{A}$ and $H, p_{i}=\left(\ln D_{A}, \ln H\right)$ for $i=1$ and 2 , and $q_{\alpha}=\left(w_{0}, w_{a}\right)$ for $\alpha=1$ and 2 .
Partial derivatives of $D_{A}$ and $H$ with respect to $w_{0}$ and $w_{a}$ are given by

$$
\begin{aligned}
\frac{\partial \ln D_{A}}{\partial w_{0}} & =-\frac{3}{2} \Omega_{\Lambda} \frac{\int_{0}^{z} \ln \left(1+z^{\prime}\right) f\left(z^{\prime}\right) g\left(z^{\prime}\right)^{-3 / 2} d z^{\prime}}{\int_{0}^{z} g\left(z^{\prime}\right)^{-1 / 2} d z^{\prime}}, \\
\frac{\partial \ln D_{A}}{\partial w_{a}} & =-\frac{3}{2} \Omega_{\Lambda} \\
& \times \frac{\int_{0}^{z}\left[\ln \left(1+z^{\prime}\right)-\frac{z^{\prime}}{1+z^{\prime}}\right] f\left(z^{\prime}\right) g\left(z^{\prime}\right)^{-3 / 2} d z^{\prime}}{\int_{0}^{z} g\left(z^{\prime}\right)^{-1 / 2} d z^{\prime}}, \\
\frac{\partial \ln H}{\partial w_{0}} & =\frac{3}{2} \Omega_{\Lambda} \ln (1+z) \frac{f(z)}{g(z)}, \\
\frac{\partial \ln H}{\partial w_{a}} & =\frac{3}{2} \Omega_{\Lambda}\left[\ln (1+z)-\frac{z}{1+z}\right] \frac{f(z)}{g(z)},
\end{aligned}
$$

where $f(z)$ and $g(z)$ are given by

$$
\begin{aligned}
& f(z)=\exp \left(3 \int_{0}^{z} \frac{1+w_{0}+w_{a} \frac{z^{\prime}}{1+z^{\prime}}}{1+z^{\prime}} d z^{\prime}\right), \\
& g(z)=\Omega_{m}(1+z)^{3}+\Omega_{\Lambda} f(z) .
\end{aligned}
$$

Figure 6 shows the derivatives as a function of $z$ between $0.5 \leqslant z \leqslant 6.5$ in two different cosmological models, $\left(w_{0}, w_{a}\right)=(-1.0,0.0)$ and $(-1.1,0.5)$. The former is the $\Lambda \mathrm{CDM}$ model, while the latter resembles the maximum likelihood values of $w_{0}$ and $w_{a}$ from the $\mathrm{WMAP}+\mathrm{BAO}+\mathrm{SN}+\mathrm{BBN}$ (Komatsu et al. 2009). The derivatives are similar for these cosmological models, and therefore we use the $\Lambda \mathrm{CDM}$ model as the fiducial model for computing the derivatives.

We add the distance information from $\mathrm{CMB}$ as

$$
\tilde{F}_{\alpha \beta}^{\text {total }}(z)=\tilde{F}_{\alpha \beta}^{\mathrm{CMB}}+\tilde{F}_{\alpha \beta}^{\mathrm{gal}}(z),
$$

where we assume that the CMB experiment yields $1 \%$ determination of the angular diameter distance out to $z=1090$, i.e., we use

$$
\tilde{F}_{\alpha \beta}^{\mathrm{CMB}}=10^{4} \frac{\partial \ln D_{A}(z=1090)}{\partial q_{\alpha}} \frac{\partial \ln D_{A}(z=1090)}{\partial q_{\beta}} .
$$

We are interested in how the BAO-only analysis compares with the full modeling. In Figure 7 we show the projected error contours on $w_{0}$ and $w_{a}$ calculated from the BAO-only analysis with the FITEX-2d and those from the full analysis at four redshift bins: $0.5 \leqslant z \leqslant 1.5,1.5 \leqslant z \leqslant 2.5,2.5 \leqslant z \leqslant 3.5$, and $3.5 \leqslant z \leqslant 4.5$. The survey area and the number of galaxies are $420 \mathrm{deg}^{2}$ and $N_{g}=2.9 \times 10^{6}$ for all redshift bins. From the BAO-only analysis we find $\left(\Delta w_{0}, \Delta w_{a}\right)=(0.29,1.26)$, $(0.38,1.39),(0.55,1.92)$, and $(0.91,3.18)$, whereas from the full modeling we find $\left(\Delta w_{0}, \Delta w_{a}\right)=(0.09,0.27),(0.06,0.17)$, $(0.09,0.35)$, and $(0.17,0.68)$, for $0.5 \leqslant z \leqslant 1.5,1.5 \leqslant z \leqslant 2.5$, $2.5 \leqslant z \leqslant 3.5$, and $3.5 \leqslant z \leqslant 4.5$, respectively.

We therefore conclude that the full analysis yields much better constraints on $w_{0}$ and $w_{a}$ than the BAO-only analysis.

\section{CONCLUSION}

In this paper, we have developed a method, called the FITEX$2 \mathrm{~d}$ method, to extract the two-dimensional phases of BAOs from galaxy power spectra in redshift space. Our model builds on and extends the existing one-dimensional algorithm, called FITEX, developed by Koehler et al. (2007). 


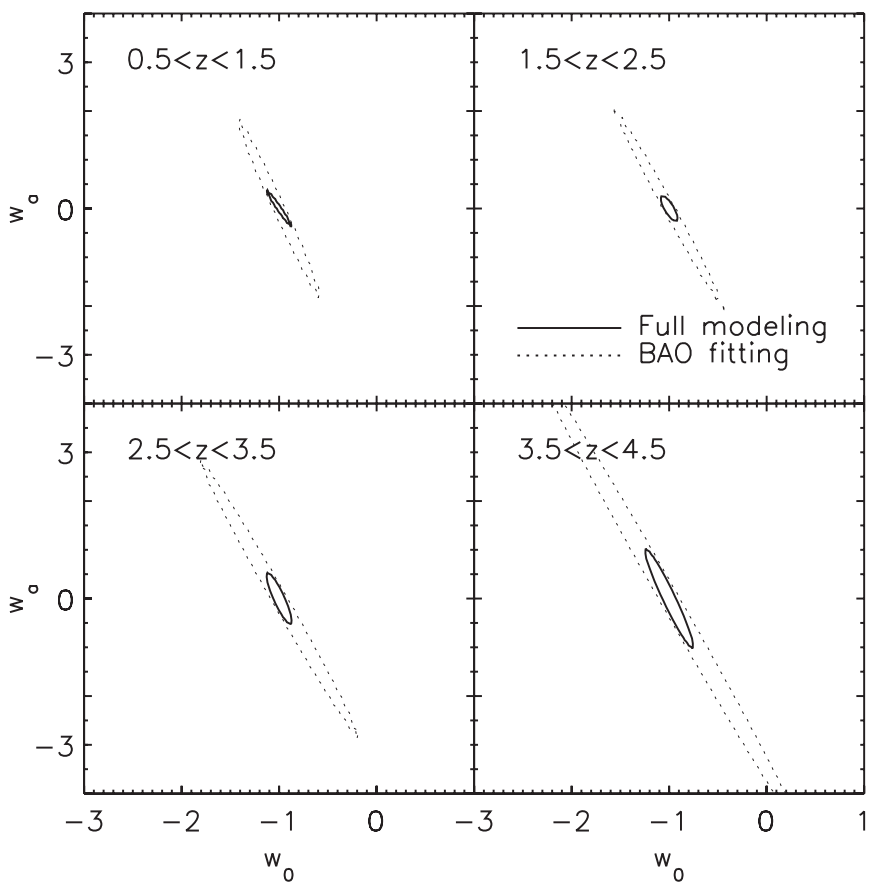

Figure 7. Projected $68 \%$ constraints on the dark energy parameters, $w_{0}$ and $w_{a}$ : the BAO fitting with the FITEX-2d method (dotted) vs. the full modeling (solid). For both cases, we use the power spectrum up to $k_{\max }=0.40 \mathrm{~h} \mathrm{Mpc}^{-1}$, and we assume that the $\mathrm{CMB}$ experiment measures the angular diameter distance out to $z=1090$ with $1 \%$ accuracy. The survey area and the number of galaxies are $420 \mathrm{deg}^{2}$ and $N_{g}=0.755 \times 10^{6}$ for all cases. Top left: $0.5 \leqslant z \leqslant 1.5$; top right: $1.5 \leqslant z \leqslant 2.5$; bottom left: $2.5 \leqslant z \leqslant 3.5$; bottom right: $3.5 \leqslant z \leqslant 4.5$. Here, the full modeling Fisher matrix is marginalized over the overall amplitude.

Our method removes the smooth, nonoscillating component from the observed galaxy power spectrum in redshift space. The fitting function consists of the smooth one-dimensional spectrum that depends only on $k, P_{\text {smooth }}^{1 d}(k)$ given by Equation (9), multiplied by the angle-dependent function expanded in the Legendre polynomials with even multipoles. The coefficients of the Legendre polynomials contain even powers of $k$. The resulting function, given by Equation (10), is able to capture the nonoscillating part of the galaxy power spectrum well.

We have tested the FITEX-2d method using the analytical model without any noise, the Monte Carlo realizations with noise expected from the HETDEX experiment (Hill et al. 2004), and the galaxy catalogue created from the Millennium Simulation (Springel et al. 2005). In all cases the FITEX$2 \mathrm{~d}$ method yields unbiased estimates of the angular diameter distance, $D_{A}$, and the expansion rate, $H$.

However, the BAOs capture only a part of distance information encoded in the galaxy power spectrum. To exploit the distance information, especially the equality scale, $r_{H}\left(z_{\mathrm{eq}}\right)$, we have explored the constraints on $D_{A}$ and $H$ from the full modeling of the galaxy power spectrum in redshift space. Provided that three key nonlinearities (nonlinear matter clustering, nonlinear galaxy bias, and nonlinear redshift space distortion) are under control, we find that the full modeling yields the constraints that are better than the BAO-only analysis by more than a factor of 2 both in $D_{A}$ and $H$, and the dark energy parameters such as $w_{0}$ and $w_{a}$.

While the effects of nonlinear matter clustering (Jeong \& Komatsu 2006; Crocce \& Scoccimarro 2008; Matarrese \& Pietroni 2007; Taruya \& Hiramatsu 2008; Valageas 2007;
Matsubara 2008; McDonald 2007) and nonlinear galaxy bias (Jeong \& Komatsu 2009) are being understood in the weakly nonlinear regime that is relevant to the future galaxy surveys, the effects of nonlinear redshift space distortion are poorly understood. While the FITEX-2d method that we have developed in this paper are useful for obtaining robust constraints on $D_{A}$ and $H$, hence the dark energy properties, one must understand nonlinear redshift space distortion to fully exploit the full information content of the galaxy power spectrum in redshift space. We would then be able to reduce the errors in $D_{A}$ and $H$ by more than a factor of 2 .

We thank the anonymous referee for illuminating comments on the correlation coefficients, which motivated our doing more thorough analysis. This material is based in part upon work supported by the Texas Advanced Research Program under Grant No. 003658-0005-2006, by NASA grants NNX08AM29G and NNX08AL43G, and by NSF grant AST-0807649. E.K. acknowledges support from an Alfred P. Sloan Research Fellowship. The Millennium Simulation databases used in this paper and the Web application providing online access to them were constructed as part of the activities of the German Astrophysical Virtual Observatory.

\section{APPENDIX}

\section{FISHER MATRIX CODE}

In this Appendix, we describe what we have implemented in our Fisher matrix code, which is publicly available as a part of "Cosmology Routine Library (CRL)," developed by one of the authors (E.K.). This code includes the nonlinear matter power spectrum in both real and redshift space, as well as marginalization over the amplitude, the linear redshift space distortion, the velocity dispersion of FoGs, the primordial tilt and running index. In the future release we plan to include nonlinear galaxy bias and primordial non-Gaussianity.

\section{A.1. Basics}

A simple, approximate formula of the Fisher matrix for galaxy survey is given by (e.g., Seo \& Eisenstein 2003)

$F_{i j}=\int_{0}^{k_{\max }} \frac{4 \pi k^{2} d k}{(2 \pi)^{3}} \int_{0}^{1} d \mu \frac{\partial \ln P_{g}(k, \mu)}{\partial \theta_{i}} \frac{\partial \ln P_{g}(k, \mu)}{\partial \theta_{j}} w(k, \mu)$,

where $P_{g}(k, \mu)$ is the galaxy survey power spectrum calculated theoretically as a function of parameters, $\theta_{i}$ are the parameters to be extracted from the data, and $w(k, \mu)$ is a function given by

$$
w(k, \mu) \equiv \frac{1}{2}\left[\frac{n_{g} P_{g}(k, \mu)}{1+n_{g} P_{g}(k, \mu)}\right] V_{\text {survey }}
$$

Here, $n_{g}$ and $V_{\text {survey }}$ are the number density of galaxies and the volume of survey, respectively.

In linear theory, $P_{g}(k, \mu)$ is given by

$$
P_{g}(k, \mu)=b_{1}^{2} R\left(\mu^{2}\right) P^{\text {linear }}(k),
$$

where $b_{1}$ is the scale independent linear bias factor, $P^{\text {linear }}(k)$ is the linear matter power spectrum, and $R\left(\mu^{2}\right)$ describes the linear redshift space distortion effect (Kaiser effect):

$$
\begin{gathered}
R\left(\mu^{2}\right) \equiv\left(1+\beta \mu^{2}\right)^{2} \\
\beta=(d \ln D / d \ln a) / b_{1},
\end{gathered}
$$

where $D$ is the growth factor of the linear density fluctuations, and $a$ is the scale factor. 


\section{A.2. Derivatives}

To calculate the logarithmic derivatives of $P(k)$ in Equation (A1), let us write down the nonlinear galaxy power spectrum (with linear bias) as (Equation 21):

$$
\begin{aligned}
P_{g}(k, \mu)= & b_{1}^{2}\left[P_{\delta \delta}(k)+2 \beta \mu^{2} P_{\delta \theta}(k)+\beta^{2} \mu^{4} P_{\theta \theta}(k)\right] \\
& \times \frac{1}{1+f^{2} k^{2} \mu^{2} \tilde{\sigma}_{v}^{2}} .
\end{aligned}
$$

We compute the derivatives with respect to the following seven parameters: the angular diameter distance, $D_{A}$, the Hubble expansion rate, $H$, the overall amplitude of the galaxy power spectrum, $A$, the linear redshift space distortion factor, $\beta \equiv$ $f / b_{1}$, the velocity dispersion with an empirically calibrated fudge factor, $\tilde{\sigma}_{v}^{2}$, the tilt of the primordial power spectrum, $n_{s}$, and the running index, $\alpha_{s}\left(P_{\text {ini }} \propto k^{n_{s}+1 / 2 \alpha_{s} \ln \left[k / k_{\text {pivot }}\right]}\right)$. We choose the convention such that

$$
\left(\theta_{1}, \theta_{2}, \theta_{3}, \theta_{4}, \theta_{5}, \theta_{6}, \theta_{7}\right)=\left(\ln D_{A}, \ln H, \ln A, \beta, \tilde{\sigma}_{v}^{2}, n_{s}, \alpha_{s}\right)
$$

The derivatives with respect to $\ln A, \beta, \tilde{\sigma}_{v}^{2}, n_{s}$, and $\alpha_{s}$ are easy to evaluate. They are given by

$$
\begin{gathered}
\frac{\partial \ln P_{g}(k, \mu)}{\partial \ln A}=1, \\
\frac{\partial \ln P_{g}(k, \mu)}{\partial \beta}=\frac{2 \mu^{2} P_{\delta \theta}(k)+2 \beta \mu^{4} P_{\theta \theta}(k)}{P_{\delta \delta}(k)+2 \beta \mu^{2} P_{\delta \theta}(k)+\beta^{2} \mu^{4} P_{\theta \theta}(k)}, \\
\frac{\partial \ln P_{g}(k, \mu)}{\partial \tilde{\sigma}_{v}^{2}}=-\frac{f^{2} k^{2} \mu^{2}}{1+f^{2} k^{2} \mu^{2} \tilde{\sigma}_{v}^{2}}, \\
\frac{\partial \ln P_{g}(k, \mu)}{\partial n_{s}}=\frac{\partial \ln P_{\text {ini }}(k)}{\partial n_{s}}=\ln k, \\
\frac{\partial \ln P_{g}(k, \mu)}{\partial \alpha_{s}}=\frac{\partial \ln P_{i n i}(k)}{\partial \alpha_{s}}=\frac{1}{2}\left[\ln \left(\frac{k}{k_{\text {pivot }}}\right)\right]^{2} .
\end{gathered}
$$

We compute the derivatives with respect to $\ln D_{A}$ and $\ln H$ in a two-step process. First, we write

$$
\begin{aligned}
\frac{\partial \ln P_{g}(k, \mu)}{\partial \ln D_{A}}= & \frac{\partial \ln P_{g}(k, \mu)}{\partial \ln k} \frac{\partial \ln k}{\partial \ln D_{A}} \\
& +\frac{\partial \ln P_{g}(k, \mu)}{\partial \mu^{2}} \frac{\partial \mu^{2}}{\partial \ln D_{A}}, \\
\frac{\partial \ln P_{g}(k, \mu)}{\partial \ln H}= & \frac{\partial \ln P_{g}(k, \mu)}{\partial \ln k} \frac{\partial \ln k}{\partial \ln H} \\
& +\frac{\partial \ln P_{g}(k, \mu)}{\partial \mu^{2}} \frac{\partial \mu^{2}}{\partial \ln H},
\end{aligned}
$$

where

$$
\begin{gathered}
\frac{\partial \ln k}{\partial \ln D_{A}}=1-\mu^{2}, \\
\frac{\partial \ln k}{\partial \ln H}=-\mu^{2}, \\
\frac{\partial \mu^{2}}{\partial \ln D_{A}}=-2 \mu^{2}\left(1-\mu^{2}\right),
\end{gathered}
$$

$$
\begin{gathered}
\frac{\partial \mu^{2}}{\partial \ln H}=-2 \mu^{2}\left(1-\mu^{2}\right) \\
\frac{\partial \ln P_{g}(k, \mu)}{\partial \mu^{2}}=\frac{2 \beta P_{\delta \theta}(k)+2 \beta^{2} \mu^{2} P_{\theta \theta}(k)}{P_{\delta \delta}(k)+2 \beta \mu^{2} P_{\delta \theta}(k)+\beta^{2} \mu^{4} P_{\theta \theta}(k)} \\
-\frac{f^{2} k^{2} \tilde{\sigma}_{v}^{2}}{1+f^{2} k^{2} \mu^{2} \tilde{\sigma}_{v}^{2}} .
\end{gathered}
$$

Finally, we need to know the "effective spectral index," $n_{\text {eff }}(k, \mu)$, given by

$$
n_{\mathrm{eff}}(k, \mu) \equiv \frac{\partial \ln P_{g}(k, \mu)}{\partial \ln k},
$$

or explicitly

$$
\begin{aligned}
n_{\mathrm{eff}}(k, \mu) & \\
= & \frac{P_{\delta \delta}(k) n_{\delta \delta}(k)+2 \beta \mu^{2} P_{\delta \theta}(k) n_{\delta \theta}(k)+\beta^{2} \mu^{4} P_{\theta \theta}(k) n_{\theta \theta}(k)}{P_{\delta \delta}(k)+2 \beta \mu^{2} P_{\delta \theta}(k)+\beta^{2} \mu^{4} P_{\theta \theta}(k)} \\
& -\frac{2 f^{2} k^{2} \mu^{2} \tilde{\sigma}_{v}^{2}}{1+f^{2} k^{2} \mu^{2} \tilde{\sigma}_{v}^{2}}
\end{aligned}
$$

where

$$
\begin{aligned}
& n_{\delta \delta}(k) \equiv \frac{\partial \ln P_{\delta \delta}(k)}{\partial \ln k}, \\
& n_{\delta \theta}(k) \equiv \frac{\partial \ln P_{\delta \theta}(k)}{\partial \ln k}, \\
& n_{\theta \theta}(k) \equiv \frac{\partial \ln P_{\theta \theta}(k)}{\partial \ln k} .
\end{aligned}
$$

\section{A.3. Correlation Coefficients}

In this subsection, we explore the behavior of the crosscorrelation coefficient between $D_{A}$ and $H$ in various cases. In particular, we focus on the effect of the marginalization over the overall amplitude with (Section A.3.2) and without (Section A.3.1) the additional marginalization over the redshift space distortion.

\section{A.3.1. No Redshift Space Distortion, $\beta=0$}

Let us evaluate the Fisher matrices in the limit that the redshift space distortion is absent, i.e., $\beta=0$. In this limit, the weighting function in Equation (A1) and the effective spectral index in Equation (A19) become independent of $\mu$, i.e., $w(k, \mu) \rightarrow w(k)$ and $n_{\text {eff }}(k, \mu) \rightarrow n_{\text {eff }}(k)$. We obtain

$$
F_{11}=\int_{k_{\min }}^{k_{\max }} \frac{k^{2} d k}{2 \pi^{2}}\left[n_{\mathrm{eff}}(k)\right]^{2} w(k) \int_{0}^{1} d \mu\left(1-\mu^{2}\right)^{2}
$$

$$
F_{12}=\int_{k_{\min }}^{k_{\max }} \frac{k^{2} d k}{2 \pi^{2}}\left[n_{\mathrm{eff}}(k)\right]^{2} w(k) \int_{0}^{1} d \mu\left(1-\mu^{2}\right)\left(-\mu^{2}\right)
$$

$$
F_{13}=\int_{k_{\min }}^{k_{\max }} \frac{k^{2} d k}{2 \pi^{2}} n_{\mathrm{eff}}(k) w(k) \int_{0}^{1} d \mu\left(1-\mu^{2}\right)
$$




$$
\begin{gathered}
F_{22}=\int_{k_{\min }}^{k_{\max }} \frac{k^{2} d k}{2 \pi^{2}}\left[n_{\mathrm{eff}}(k)\right]^{2} w(k) \int_{0}^{1} d \mu\left(-\mu^{2}\right)^{2} \\
F_{23}=\int_{k_{\min }}^{k_{\max }} \frac{k^{2} d k}{2 \pi^{2}} n_{\mathrm{eff}}(k) w(k) \int_{0}^{1} d \mu\left(-\mu^{2}\right) \\
F_{33}=\int_{k_{\min }}^{k_{\max }} \frac{k^{2} d k}{2 \pi^{2}} w(k) \int_{0}^{1} d \mu .
\end{gathered}
$$

Now, in order to understand the effect of the structure of $n_{\mathrm{eff}}$, let us assume that the galaxy power spectrum is a pure power-law, i.e., $n_{\text {eff }}(k)=n$ and $n$ is the independent of $k$. In this limit, we obtain

$$
F_{i j}=\bar{w}\left(\begin{array}{ccc}
\frac{8 n^{2}}{15} & -\frac{2 n^{2}}{15} & \frac{2 n}{3} \\
-\frac{2 n^{2}}{15} & \frac{n^{2}}{5} & -\frac{n}{3} \\
\frac{2 n}{3} & -\frac{n}{3} & 1
\end{array}\right)
$$

where $\bar{w} \equiv \int \frac{k^{2} d k}{2 \pi^{2}} w(k)$.

The marginalized errors of parameters and the correlation coefficients are computed from the inverse of the Fisher matrix. However, one can show that the matrix given in Equation (A30) is singular. In other words, $D_{A}$ and $H$ are completely degenerate with the amplitude for a power-law power spectrum. This result shows that only the departure of the power spectrum from a pure power-law, i.e., the existence of characteristic scales, can break the degeneracy between $D_{A}$ and $H$, and $A$. These scales are often called the "standard rulers."

To understand the structure of the Fisher matrix in Equation (A30) better, let us add small perturbations, $\epsilon>0$, to the diagonal elements, and invert the matrix. The result is

$$
\left(F^{-1}\right)_{i j}=\frac{1}{\bar{w}}\left(\begin{array}{ccc}
\frac{1}{\left(2+n^{2}\right) \epsilon} & -\frac{1}{\left(2+n^{2}\right) \epsilon} & -\frac{n}{\left(2+n^{2}\right) \epsilon} \\
-\frac{1}{\left(2+n^{2}\right) \epsilon} & \frac{1}{\left(2+n^{2}\right) \epsilon} & \frac{n}{\left(2+n^{2}\right) \epsilon} \\
-\frac{n}{\left(2+n^{2}\right) \epsilon} & \frac{n}{\left(2+n^{2}\right) \epsilon} & \frac{n^{2}}{\left(2+n^{2}\right) \epsilon}
\end{array}\right)+\mathcal{O}\left(\epsilon^{0}\right) .
$$

We find that the correlation coefficient between $D_{A}$ and $H$ is

$$
r_{12} \equiv \frac{\left(F^{-1}\right)_{12}}{\sqrt{\left(F^{-1}\right)_{11}\left(F^{-1}\right)_{22}}} \rightarrow-1
$$

as $\epsilon \rightarrow 0$. Therefore, $\ln D_{A}$ and $\ln H$ are totally anti-correlated, which implies that, although we cannot determine $\ln D_{A}$ and $\ln H$ simultaneously, we can determine $\ln D_{A}+\ln H=$ $\ln \left(D_{A} H\right)$, even for a power-law power spectrum. ${ }^{8}$ This is known as the Alcock-Paczyński (AP) test (Alcock \& Paczynski 1979).

There is a special case in which the covariance between $A$ and $D_{A}$ or $H$ may be ignored. One may imagine the situation where $n_{\text {eff }}(k)$ depends upon $k$ such that $A$ is uncorrelated with $D_{A}$ or $H$. For example, if $n_{\text {eff }}(k)$ oscillates about zero, then $\int_{k_{\min }}^{k_{\max }} \frac{k^{2} d k}{2 \pi^{2}} n_{\text {eff }}(k) w(k)$ would be small compared with $\int_{k_{\min }}^{k_{\max }} \frac{k^{2} d k}{2 \pi^{2}}\left[n_{\mathrm{eff}}(k)\right]^{2} w(k)$ or $\int_{k_{\min }}^{k_{\max }} \frac{k^{2} d k}{2 \pi^{2}} w(k)$. Therefore, $F_{13}$ and $F_{23}$ may be ignored, making $A$ decorrelated with $D_{A}$ and $H$. In this case, the Fisher matrix is a two-by-two matrix given by

$$
F_{i j}=\bar{w} n^{2}\left(\begin{array}{cc}
\frac{8}{15} & -\frac{2}{15} \\
-\frac{2}{15} & \frac{1}{5}
\end{array}\right) .
$$

\footnotetext{
8 The other cross-correlation coefficients are $r_{13} \rightarrow \mp 1$ and $r_{23} \rightarrow \pm 1$ for $n>0$ and $n<0$, respectively.
}

The inverse of this matrix is then

$$
\left(F^{-1}\right)_{i j}=\frac{1}{\bar{w} n^{2}}\left(\begin{array}{cc}
\frac{9}{4} & \frac{3}{2} \\
\frac{3}{2} & 6
\end{array}\right) .
$$

The correlation coefficient between $D_{A}$ and $H$ is thus given by

$$
r_{12}=\frac{3 / 2}{\sqrt{9 / 4 \times 6}}=\frac{1}{\sqrt{6}} \simeq 0.408 .
$$

This result has been derived by Seo \& Eisenstein (2007), and justifies the use of BAOs as a way to measure $D_{A}$ and $H$ with a correlation coefficient of 0.408 .

From these studies we are led to the following conclusions:

1. When the information is dominated by BAOs, the correlation coefficient between $D_{A}$ and $H$ is $r_{12} \simeq 0.408$. The amplitude of the BAOs contributes little to the errors on $D_{A}$ and $H$, as the amplitude information is de-correlated with $D_{A}$ and $H$.

2. When the information is dominated by the AP test, $r_{12} \simeq$ -1 .

3. In reality, as we have shown in this paper, BAOs contribute less than the overall shape of the power spectrum. Also, the shape of the power spectrum is not exactly a power-law. As a result, the correlation coefficient from the full analysis is usually negative (or small positive), but always greater than -1 (see Table 1).

\section{A.3.2. With Redshift Space Distortion, $\beta>0$}

Next, let us consider the case where the redshift space distortion cannot be ignored. In this case, we see from Equations (A2) and (A20) that the weighting function, $w(k, \mu)$, and the effective spectral index, $n_{\text {eff }}(k, \mu)$, are no longer independent of $\mu$. The analytical treatment is also possible for this case, although the results are too complicated to be useful. We therefore report on the numerical results.

Here, we choose the survey parameters given in Section 3.2 with the nonlinear power spectrum of Equation (21). The results from the numerical calculations of the Fisher matrix are given in Table 1. We find that the marginalization over the amplitude information, and that over the amplitude and the shape of the primordial power spectrum (i.e., $n_{s}$ and $\alpha_{s}$ ) give the crosscorrelation close to -1 ; thus, one relies on the AP test. The marginalization over the amplitude and the linear redshift space distortion (i.e., $\beta$ ) drive the cross-correlation towards zero, as the AP test no longer works when the linear redshift space distortion is marginalized over. However, in both cases the errors in the combined one-dimensional distance scale, $R$, are about the same. In other words, while one changes the orientation of the ellipse, the area is approximately preserved.

In summary, when the amplitude information is marginalized over, the information is mostly coming from the dependence of $P(k, \mu)$ on $\mu^{2}$, which yields a constraint on $D_{A H}$ via the AP test, while when both the amplitude and the linear redshift space distortion are marginalized, the most information is coming from the standard rulers, which can constrain $D_{A}$ and $H$ separately, driving the cross-correlation towards zero.

Finally, in Figure 8 we show how different choices of marginalization over parameters influence the error contours of $w_{0}$ and $w_{a}:\left(\Delta w_{0}, \Delta w_{a}\right)=(0.08,0.27),(0.08,0.30)$, 


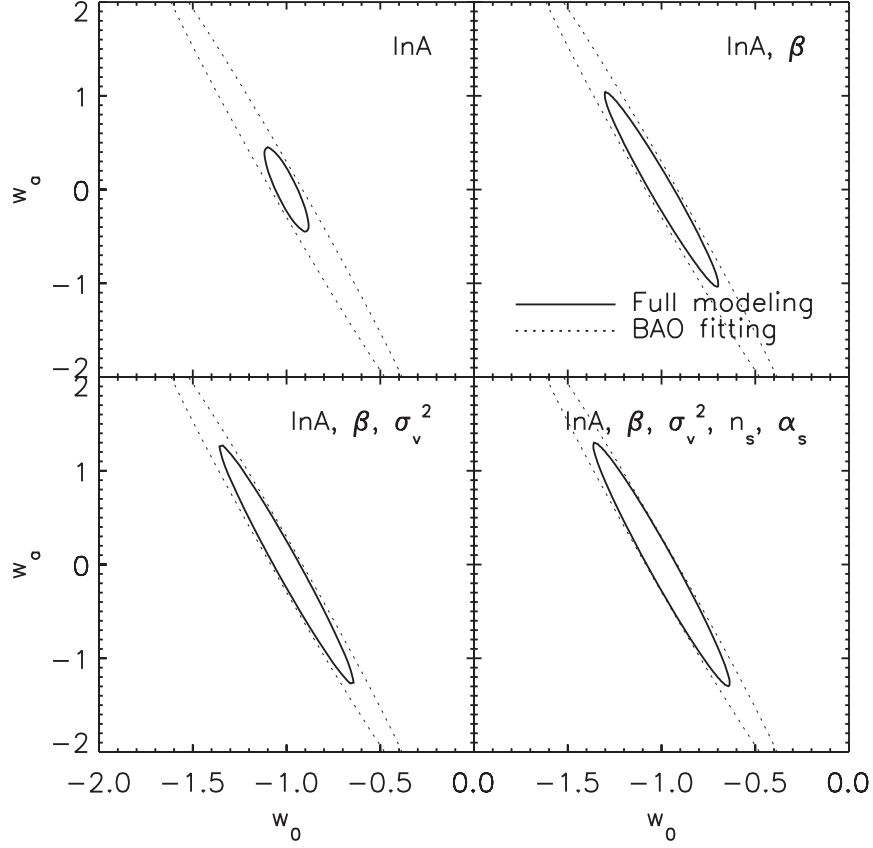

Figure 8. Projected $68 \%$ constraints on the dark energy parameters, $w_{0}$ and $w_{a}$. The full modeling (solid) marginalized over different combinations of parameters as well as the BAO-only analysis (dotted) are shown. For all cases, we use the power spectrum up to $k_{\max }=0.40 h \mathrm{Mpc}^{-1}$, and we assume that the $\mathrm{CMB}$ experiment measures the angular diameter distance out to $z=1090$ with $1 \%$ accuracy. The survey area and the number of galaxies are $420 \mathrm{deg}^{2}$ and $N_{g}=0.755 \times 10^{6}$, and the redshift range is $1.9 \leqslant z \leqslant 3.5$ for all cases. (Top Left) marginalized over $\ln A$, (top right) marginalized over $\ln A, \beta$, (bottom left) marginalized over $\ln A, \beta$ and $\tilde{\sigma}_{v}^{2}$, (bottom right) marginalized over $\ln A, \beta, \tilde{\sigma}_{v}^{2}$, $n_{s}$ and $\alpha_{s}$.

$(0.24,0.85)$, and $(0.24,0.86)$, for the cases of no marginalization, marginalization over $\ln A$, marginalization over $\ln A, \beta$ and $\tilde{\sigma}_{v}^{2}$, and marginalization over $\ln A, \beta, \tilde{\sigma}_{v}^{2}, n_{s}$, and $\alpha_{s}$, respectively.

\section{A.4. User's Guide}

When using the Fisher matrix code, one may choose the form of the model galaxy power spectrum from:

1. Linear power spectrum with the linear redshift space distortion (Kaiser effect);

2. Nonlinear power spectrum from the third-order perturbation theory with the linear redshift space distortion;

3. Nonlinear power spectrum from the third-order perturbation theory with the nonlinear redshift space distortion given by Equation (71) of Scoccimarro (2004); or

4. Nonlinear power spectrum from the third-order perturbation theory with the nonlinear redshift space distortion given by Equation (21).

Next, specify the number of parameters one wishes to marginalize over, and then choose the parameters from $\ln A$, $\beta, \tilde{\sigma}_{v}^{2}, n_{s}$, and $\alpha_{s}$.

A given galaxy survey can be sliced up into multiple redshift bins. After entering the survey area in units of $\mathrm{deg}^{2}$, one is asked to enter the following parameters at each redshift bin: the redshift range $\left(z_{\min }<z<z_{\max }\right)$, the number of galaxies in units of millions in the bin, $b_{1}, k_{\max }$ in units of $h \mathrm{Mpc}^{-1}$, and the redshift error in units of $\mathrm{km} \mathrm{s}^{-1}$.

The linear power spectrum at $z=30$ has been precomputed using the CAMB code (Lewis et al. 2000) for the maximum likelihood parameters given in Table 1 of Komatsu et al. (2009) ("WMAP+BAO+SN"). The ingredients of the nonlinear power spectra, $P_{\delta \delta}, P_{\delta \theta}$, and $P_{\theta \theta}$, have been precomputed from the linear spectrum at $z=30$. These spectra are then evolved to a specified redshift by the appropriate growth factor obtained by solving the differential equation given in Equation (76) of Komatsu et al. (2009).

Finally, the code yields the errors on $\ln D_{A}, \ln H, r_{D_{A}, H}$, and $\ln R$ (see Equation 29 for the definition of the error in the combined distance scale, $R$ ).

\section{REFERENCES}

Afshordi, N., Loh, Y.-S., \& Strauss, M. A. 2004, Phys. Rev. D, 69, 083524 Alcock, C., \& Paczynski, B. 1979, Nature, 281, 358

Angulo, R. E., Baugh, C. M., Frenk, C. S., \& Lacey, C. G. 2008, MNRAS, 383, 755

Ballinger, W. E., Peacock, J. A., \& Heavens, A. F. 1996, MNRAS, 282, 877

Benson, A. J., Bower, R. G., Frenk, C. S., Lacey, C. G., Baugh, C. M., \& Cole, S. 2003, ApJ, 599, 38

Blake, C., \& Glazebrook, K. 2003, ApJ, 594, 665

Boughn, S., \& Crittenden, R. 2004, Nature, 427, 45

Bower, R. G., Benson, A. J., Malbon, R., Helly, J. C., Frenk, C. S., Baugh, C M., Cole, S., \& Lacey, C. G. 2006, MNRAS, 370, 645

Chevallier, M., \& Polarski, D. 2001, Int. J. Mod. Phys., D10, 213

Cole, S., Lacey, C. G., Baugh, C. M., \& Frenk, C. S. 2000, MNRAS, 319, 168

Cole, S., et al. 2005, MNRAS, 362, 505

Copeland, E. J., Sami, M., \& Tsujikawa, S. 2006, Int. J. Mod. Phys., D15, 1753

Crocce, M., \& Scoccimarro, R. 2008, Phys. Rev. D, 77, 023533

Davis, M., \& Peebles, P. J. E. 1983, ApJ, 267, 465

Dunkley, J., et al. 2009, ApJS, 180, 306

Eisenstein, D. J., Hu, W., \& Tegmark, M. 1999, ApJ, 518, 2

Eisenstein, D. J., Seo, H.-J., \& White, M. 2007, ApJ, 664, 660

Eisenstein, D. J., et al. 2005, ApJ, 633, 560

Evrard, A. E., et al. 2002, ApJ, 573, 7

Fosalba, P., \& Gaztañaga, E. 2004, MNRAS, 350, L37

Fosalba, P., Gaztañaga, E., \& Castander, F. J. 2003, ApJ, 597, L89

Fry, J. N., \& Gaztanaga, E. 1993, ApJ, 413, 447

Heavens, A. F., Matarrese, S., \& Verde, L. 1998, MNRAS, 301, 797

Hill, G. J., Gebhardt, K., Komatsu, E., \& MacQueen, P. J. 2004, AIP Conf. Ser., 743,224

Hinshaw, G., et al. 2009, ApJS, 180, 225

Hu, W., \& Haiman, Z. 2003, Phys. Rev. D, 68, 063004

Hütsi, G. 2006, A\&A, 449, 891

Jeong, D., \& Komatsu, E. 2006, ApJ, 651, 619

Jeong, D., \& E., Komatsu 2009, ApJ, 691, 569

Kaiser, N. 1987, MNRAS, 227, 1

Koehler, R. S., Schuecker, P., \& Gebhardt, K. 2007, A\&A, 462, 7

Komatsu, E., et al. 2009, ApJS, 180, 330

Kowalski, M., et al. 2008, ApJ, 686, 749

Lewis, A., Challinor, A., \& Lasenby, A. 2000, ApJ, 538, 473

Linder, E. V. 2003, Phys. Rev. Lett., 90, 091301

Matarrese, S., \& Pietroni, M. 2007, J. Cosmol. Astropart. Phys., 6, 26

Matsubara, T. 2008, Phys. Rev. D, 77, 063530

McDonald, P. 2006, Phys. Rev. D, 74, 103512

McDonald, P. 2007, Phys. Rev. D, 75, 043514

Nishimichi, T., et al. 2007, PASJ, 59, 1049

Nolta, M. R., et al. 2004, Astrophys. J., 608, 10

Okumura, T., Matsubara, T., Eisenstein, D. J., Kayo, I., Hikage, C., Szalay, A. S., \& Schneider, D. P. 2008, ApJ, 676, 889

Padmanabhan, N., \& White, M. 2008, Phys. Rev. D, 77, 123540

Peebles, P. J. E. 1976, Ap\&SS, 45, 3

Percival, W. J., Cole, S., Eisenstein, D. J., Nichol, R. C., Peacock, J. A., Pope, A. C., \& Szalay, A. S. 2007, MNRAS, 381, 1053

Perlmutter, S., et al. 1999, ApJ, 517, 565

Riess, A. G., et al. 1998, AJ, 116, 1009

Sanchez, A. G., Baugh, C. M., \& Angulo, R. 2008, MNRAS, 390, 1470

Scoccimarro, R. 2004, Phys. Rev. D, 70, 083007

Sefusatti, E., \& Komatsu, E. 2007, Phys. Rev. D, 76, 083004 
Seo, H.-J., \& Eisenstein, D. J. 2003, ApJ, 598, 720

Seo, H.-J., \& Eisenstein, D. J. 2005, Astrophys. J., 633, 575

Seo, H.-J., \& Eisenstein, D. J. 2007, ApJ, 665, 14

Seo, H.-J., Siegel, E. R., Eisenstein, D. J., \& White, M. 2008, ApJ, 686, 13

Smith, R. E., Scoccimarro, R., \& Sheth, R. K. 2008, Phys. Rev. D, 77, 043525
Springel, V., et al. 2005, Nature, 435, 629

Taruya, A., \& Hiramatsu, T. 2008, ApJ, 674, 617

Valageas, P. 2007, A\&A, 465, 725

Wagner, C., Müller, V., \& Steinmetz, M. 2008, A\&A, 487, 63

Weinberg, S. 2008, Cosmology (Oxford: Oxford Univ. Press)

Yamamoto, K., Bassett, B. A., \& Nishioka, H. 2005, Phys. Rev. Lett., 94, 051301 